\title{
Fermionic CFTs and classifying algebras
}

\author{
Ingo Runkel ${ }^{a, b}$ and Gérard M.T. Watts ${ }^{b}$ \\ ${ }^{a}$ Fachbereich Mathematik, Universität Hamburg, \\ Bundesstraße 55, Hamburg 20146, Germany \\ ${ }^{b}$ Department of Mathematics, King's College London, \\ Strand, London WC2R 2LS, U.K. \\ E-mail: ingo.runkel@uni-hamburg.de, gerard.watts@kcl.ac.uk
}

ABSTRACT: We study fermionic conformal field theories on surfaces with spin structure in the presence of boundaries, defects, and interfaces. We obtain the relevant crossing relations, taking particular care with parity signs and signs arising from the change of spin structure in different limits. We define fermionic classifying algebras for boundaries, defects, and interfaces, which allow one to read off the elementary boundary conditions, etc.

As examples, we define fermionic extensions of Virasoro minimal models and give explicit solutions for the spectrum and bulk structure constants. We show how the $A$ and $D$-type fermionic Virasoro minimal models are related by a parity-shift operation which we define in general. We study the boundaries, defects, and interfaces in several examples, in particular in the fermionic Ising model, i.e. the free fermion, in the fermionic tri-critical Ising model, i.e. the first unitary $N=1$ superconformal minimal model, and in the supersymmetric Lee-Yang model, of which there are two distinct versions that are related by parity-shift.

KeYwords: Conformal Field Theory, Boundary Quantum Field Theory, Conformal and W Symmetry

ARXIV EPRINT: 2001.05055 


\section{Contents}

1 Introduction 1

2 Bulk fields in fermionic CFT 2

2.1 The topological defect $F \quad 2$

2.2 Bulk structure constants 4

2.3 Crossing symmetry constraint 5

$\begin{array}{lll}2.4 & \text { Symmetry properties of structure constants } & 6\end{array}$

$\begin{array}{lll}2.5 & \text { Parity shift of the Ramond sector } & 7\end{array}$

$\begin{array}{lll}3 & \text { Classifying algebras } & \mathbf{7}\end{array}$

3.1 Bulk-boundary OPE and boundary classifying algebra 8

3.1.1 Conventions for bulk-boundary OPE and boundary OPE 8

$\begin{array}{lll}3.1 .2 & \text { Bulk-boundary crossing relation } & 8\end{array}$

$\begin{array}{lll}\text { 3.1.3 Boundary classifying algebra: bosonic case } & 9\end{array}$

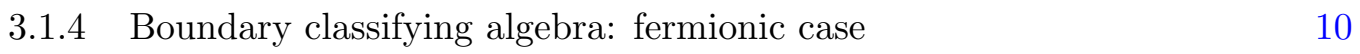

$\begin{array}{lll}3.2 & \text { Bulk-defect OPE and defect classifying algebra } & 12\end{array}$

$\begin{array}{ll}3.3 & \text { Classifying algebras in the parity shifted theory } \\ & 13\end{array}$

$\begin{array}{lll}3.3 .1 & \text { Fermionic boundary classifying algebra } & 13\end{array}$

$\begin{array}{lll}\text { 3.3.2 } & \text { Fermionic defect classifying algebra } & 14\end{array}$

4 Fermionic Virasoro minimal models $\quad \mathbf{1 5}$

$\begin{array}{lll}4.1 & \text { A-type fermionic models } & 16\end{array}$

$\begin{array}{lll}4.2 & \text { D-type fermionic models } & 17\end{array}$

5 The Ising model and the free fermion $\quad 19$

$\begin{array}{lll}5.1 \text { Bulk structure constants } & 19\end{array}$

$\begin{array}{ll}5.2 & \text { Boundary classifying algebra and boundary conditions }\end{array}$

5.3 Boundary field content and boundary states 22

$\begin{array}{lll}\text { 5.3.1 Boundary field content } & 22\end{array}$

5.3.2 Boundary states 25

5.4 Defect classifying algebra and defect operators 26

$\begin{array}{ll}\text { 5.4.1 Classifying algebra and defect conditions } & 26\end{array}$

$\begin{array}{ll}\text { 5.4.2 Defect field content and defect operators } & 27\end{array}$

5.5 Interfaces between Ising and fermionic Ising 29

6 Further Virasoro examples $\quad 29$

6.1 Fermionic tri-critical Ising model 30

6.1.1 Fermionic TCIM boundary classifying algebra 30

6.1.2 Comparison to [13, 14] 31

6.1.3 Fermionic TCIM defect classifying algebra 32

6.1.4 Fermionic TCIM interface classifying algebra 33 
A Data for the Ising and tri-critical Ising models

$\begin{array}{ll}\text { A.1 Ising data } & 37\end{array}$

$\begin{array}{lll}\text { A.2 Tri-critical Ising data } & 38\end{array}$

\section{Introduction}

In this paper we set out a way to define conformal field theories with fermions and analyse their conformal boundary conditions, defects and interfaces. Conformal field theories with fermions have been studied for a very long time, as have their boundary conditions, but in this paper we take an algebraic approach to the description of fermionic theories and spin structures. This makes an algebraic analysis of boundary conditions and defects tractable and has revealed new relations between models that had not been understood before.

The first objective is to define correlation functions of fields in fermionic conformal field theories unambiguously on surfaces with spin structures; we do this using defects as proposed in [1]. In this paper we outline this method and state our results; we will give more details and proofs in [2].

Our description of fermionic CFTs has two immediate implications. Firstly, the state space is a super-vector space, divided by the grading into fields of even and odd parity. Secondly, we need to consider the different spin structures separately as each spin structure defines a different way to put fermions on a surface consistently, so that each circular boundary, defect, and interface will have a separate description for the two possible spin structures in its neighbourhood. We obtain a consistent set of sewing constraints for theories including fermions, which incorporates the signs that arise from re-ordering of products of fermionic fields and - importantly - extra signs that arise from putting the spin structure back in a standard form in different limits. We describe this in detail in section 2. One particularly interesting result is that given a fermionic CFT, it is possible to define another fermionic CFT in which the parities (odd/even) are swapped in the Ramond sector. This can either result in the original CFT again or in a new CFT.

Having defined the bulk theories, we are able to consider their conformal boundary conditions, defects and interfaces. We show in section 3 how the bulk-boundary, bulkdefect and bulk-interface structure constants define super-algebras, which we refer to as fermionic classifying algebras, and which then allow one to identify the fundamental boundary conditions, etc., algebraically from these algebras alone. For purely bosonic theories the corresponding classifying algebras were introduced in $[3,4]$. One consequence is the natural occurrence of fermionic weight zero fields on boundaries, defects and interfaces, which are required for their consistent description. These have been known for a long time, 
for example they have been used in the coupling of the Ising model to boundary magnetic fields $[5,6]$, see also $[7,8]$ for more recent applications to boundary renormalisation group flows. Our analysis shows they are a necessary and integral part of the description of fermionic CFTs with boundaries, defects and interfaces.

We illustrate these ideas in the concrete cases of fermionic extensions of Virasoro minimal models which we define in section 4 , and for which we give an explicit solution for the bulk structure constants. As is well known, for some values of the central charge there are two or more different Virasoro minimal models labelled by pairs of Lie algebras [9]. We show that, remarkably, the fermionic extensions of the $\left(A_{m}, A_{4 n-1}\right)$ and $\left(A_{m}, D_{2 n+1}\right)$ bosonic minimal models - and in particular their bulk structure constants $[10,11]$ - are related by the parity-shift operation.

The fermionic extensions of the Virasoro minimal models include such important examples as the fermionic Ising model, i.e. the free fermion, and the fermionic tri-critical Ising model, i.e. the first unitary $N=1$ superconformal minimal model, as well as many other theories with extended symmetries. Sections 5 and 6 of the paper are taken up with exploring our results in these situations and comparing our findings with the discussions already in the literature, in particular [12-14].

\section{Bulk fields in fermionic CFT}

In this section we explain how to describe fields of a fermionic CFT. In order to include the effects of the spin structure and the bosonic/fermionic nature of fields, we introduce a special type of topological line defect; all bulk fields are then connected to one of these defects, so that we think of them as disorder fields which sit at the starting point of the specific topological defect.

We start by describing the relevant properties of the topological defect and then use these to define OPE coefficients and to obtain the crossing constraint they have to satisfy. We show that, given one solution to the crossing constraint, one can obtain another solution by shifting the Ramond sector parity and modifying the given solution by signs.

\subsection{The topological defect $F$}

The spin structure on the worldsheet of a fermionic CFT is encoded by a topological defect which we call $F$. The technical details of this procedure are given in $[1,15]$. Here we do not need the full formalism and just state the properties we will use below.

We denote by $\mathcal{H}_{F}$ the space of disorder fields that sit at the start of the topological defect $F$ (figure 1a). Since $F$ is topological, $\mathcal{H}_{F}$ carries a representation of the holomorphic and anti-holomorphic copy of the Virasoro algebra.

The space $\mathcal{H}_{F}$ is a super-vector space, that is, it is $\mathbb{Z}_{2}$-graded into an even and an odd component,

$$
\mathcal{H}_{F}=\mathcal{H}_{F}^{\mathrm{ev}} \oplus \mathcal{H}_{F}^{\text {odd }} .
$$

We refer to this grading as parity, and for a homogeneous element $\phi \in \mathcal{H}_{F}$ we write $|\phi| \in\{0,1\}$ - or just $\phi$ if no confusion can arise - for its parity. There is a second $\mathbb{Z}_{2^{-}}$ 


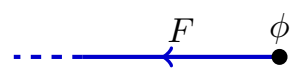

(a)

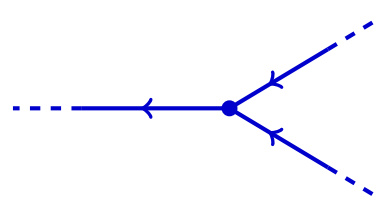

(c)

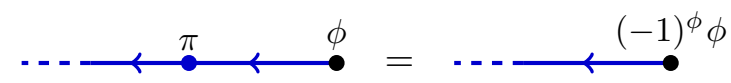

(b)

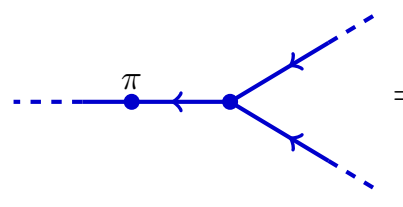

(d)

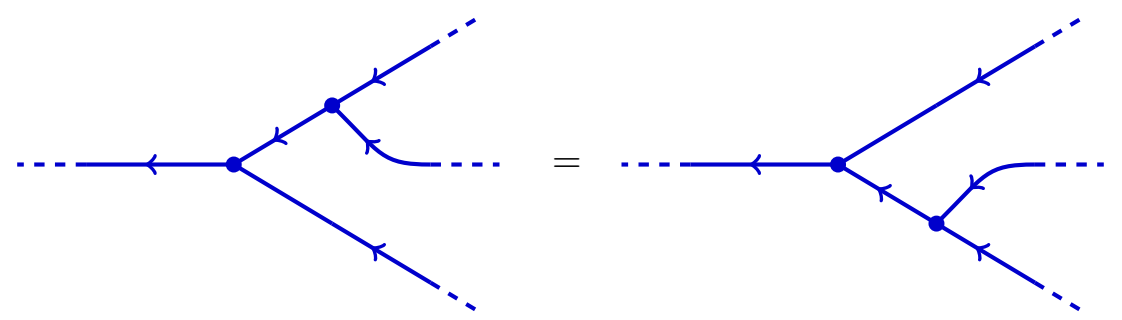

(e)

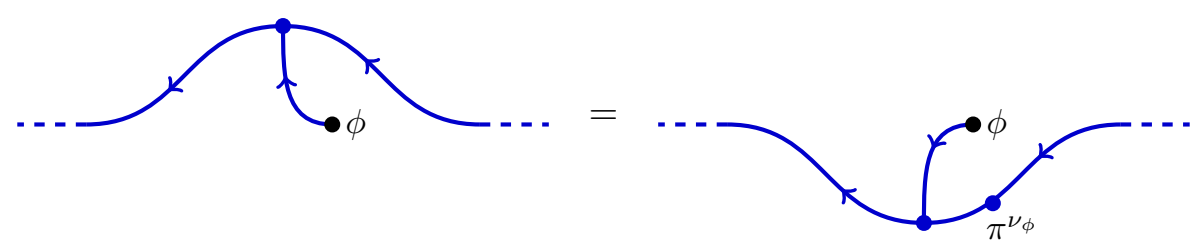

(f)

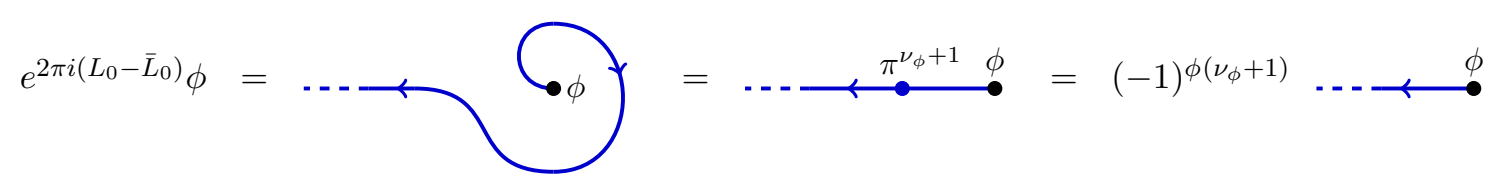

(g)

Figure 1. Properties of the topological line defect $F$. a) A field $\phi \in \mathcal{H}_{F}$ sits at the start of $F$. b) The OPE of the weight zero defect field $\pi$ and a field $\phi$ multiplies $\phi$ by \pm 1 , depending on its parity. c) The weight zero junction joining two $T$ defects into one. d) Pushing the defect field $\pi$ through the defect junction. e) Associativity relation for the junction field. f) Dragging the $F$ defect through a field $\phi$ of spin grade $\nu_{\phi}$ inserts $\pi^{\nu_{\phi}}$. g) Rotating $\phi \in \mathcal{H}_{F}^{\nu_{\phi}}$ by $2 \pi$ can be traded for an insertion of $\pi^{\nu_{\phi}+1}$. In particular, one cannot just unwind an $F$-defect around $\phi$, instead the tangent at the insertion point of $\phi$ has to remain fixed.

grading on $\mathcal{H}_{F}$ whose components are called the Neveu-Schwarz and the Ramond sector,

$$
\mathcal{H}_{F}=\mathcal{H}_{F}^{\mathrm{NS}} \oplus \mathcal{H}_{F}^{\mathrm{R}} .
$$

We will refer to this as the spin grading. It will be convenient to abbreviate $\mathcal{H}_{F}^{0}=\mathcal{H}_{F}^{\mathrm{NS}}$ and $\mathcal{H}_{F}^{1}=\mathcal{H}_{F}^{\mathrm{R}}$. Each $\mathcal{H}_{F}^{\nu}$ is still parity graded, $\mathcal{H}_{F}^{\nu}=\mathcal{H}_{F}^{\nu, \text { ev }} \oplus \mathcal{H}_{F}^{\nu, \text { odd }}$, so that altogether, $\mathcal{H}_{F}$ is $\mathbb{Z}_{2} \times \mathbb{Z}_{2}$-graded. For a field $\phi \in \mathcal{H}_{F}$ that is homogeneous with respect to the spin grading we write $\nu_{\phi} \in\{0,1\}$ for its degree. 
We will refer to fields in $\mathcal{H}_{F}$ as bulk fields of the fermionic CFT.

The defect $F$ has the following properties:

1. There is a parity even weight zero ${ }^{1}$ defect field $\pi$ on $F$ which implements parity on $F$ in the sense that the OPE of $\pi$ with a bulk field $\phi$ is $(-1)^{\phi} \phi$ (figure $1 \mathrm{~b}$ ). It will be convenient to write $\pi^{0}=1$ for the identity defect field and $\pi^{1}=\pi$.

2. There is a parity even weight zero defect junction joining two in-coming $F$-defects into an out-going $F$-defect (figure 1c). This junction commutes with $\pi$ in the sense shown in figure $1 \mathrm{~d}$, and it is associative as shown in figure $1 \mathrm{e}$.

3. Taking a bulk field $\phi$ of spin grade $\nu_{\phi}$ past an $F$-defect results in the insertion of $\pi^{\nu_{\phi}}$ as shown in figure 1f.

One can verify that the effect of a $2 \pi$-rotation of a bulk field $\phi$ can be replaced by an insertion of $\pi^{\nu_{\phi}+1}$ on the $F$-defect (figure $1 \mathrm{~g}$ ), see [1, lemma 4.7]. Thus we get

$$
e^{2 \pi i\left(L_{0}-\bar{L}_{0}\right)} \phi=(-1)^{\phi\left(\nu_{\phi}+1\right)} \phi .
$$

Denote the conformal spin of a field by $S_{\phi}:=h_{\phi}-\bar{h}_{\phi}$. The above relation implies that

$$
S_{\phi} \in \begin{cases}\mathbb{Z}+\frac{1}{2} ; & \phi \text { is a parity-odd NS-field }, \\ \mathbb{Z} ; & \text { otherwise }\end{cases}
$$

\section{$2.2 \quad$ Bulk structure constants}

To define the OPE of bulk fields, we need to fix a convention for the spin structure in a neighbourhood of the fields. In the present formalism, this is done by requiring a particular pattern of defect lines. The convention we will use is that for $x>y$ real,

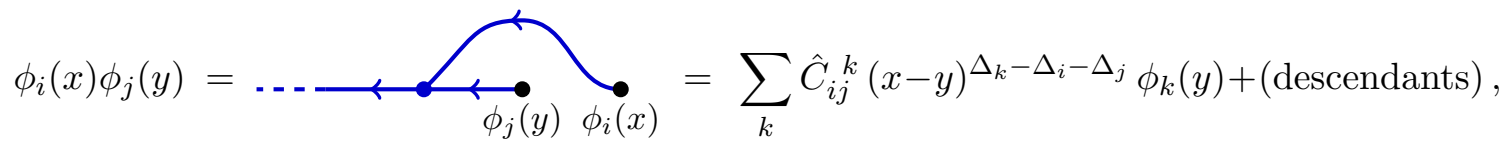

where the $\phi_{i}$ are a basis of primary fields in $\mathcal{H}_{F}$, and $\Delta_{i}=h_{i}+\bar{h}_{i}$ denotes the scaling dimension of $\phi_{i}$.

One could alternatively have chosen the convention that the $F$-defect starting at $\phi_{i}$ passes below $\phi_{j}$. According to figure 1f, the two choices are related by

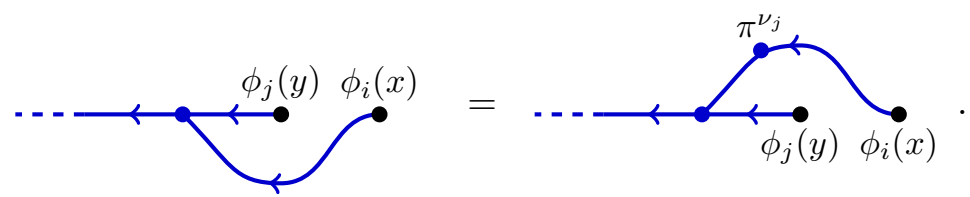

If we denote the structure constants computed in the $F$-defect-passes-below convention by $\check{C}_{i j}^{k}$, then the resulting relation is

$$
\check{C}_{i j}^{k}=(-1)^{\phi_{i} \nu_{j}} \hat{C}_{i j}^{k}
$$

In the following we will stick to the convention in (2.5).

\footnotetext{
${ }^{1}$ We use "weight zero" to mean that it behaves like a vacuum field, i.e. that it is annihilated by the translation operators $L_{-1}$ and $\bar{L}_{-1}$.
} 


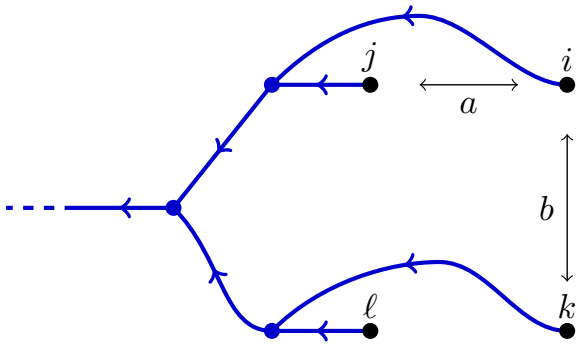

(a)

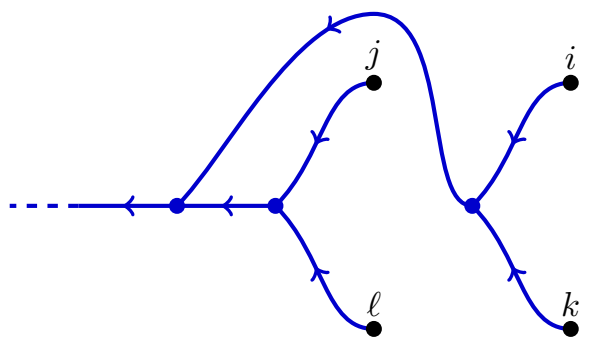

(b)

Figure 2. a) The arrangement of $F$-defects needed to substitute the OPE in the $a \rightarrow 0$ limit. b) The corresponding configuration required in the $b \rightarrow 0$ limit.

\subsection{Crossing symmetry constraint}

Consider a correlator $f(a, b)$ of four primary bulk fields $\phi_{i}, \phi_{j}, \phi_{k}, \phi_{\ell}$, inserted at positions $a+i b, i b, a, 0$ for some $a, b>0$,

$$
f(a, b)=\left\langle\phi_{i}(a+i b) \phi_{j}(i b) \phi_{k}(a) \phi_{\ell}(0)\right\rangle .
$$

Here it is understood that the $F$-defects are placed as in figure $2 \mathrm{a}$, and that the ordering relevant for the parity signs is radial ordering.

For simplicity, in the derivation of the crossing constraint (and in that of similar constraints below) we restrict ourselves to theories which are rational with respect to the Virasoro symmetry. Extended chiral algebras can be treated in the same way, but one has to account for two complications. Firstly, the fusing matrices will in general carry multiplicity labels. Secondly, the leading contribution in the OPE of two primary fields (with respect to the extended symmetry) may be a descendent field, which makes the definition of the OPE coefficients more involved.

The defect arrangement in figure 2a is such that in the $a \rightarrow 0$ limit, we can substitute the bulk OPE right away. Hence in this limit we simply have

$$
f(a, b) \underset{a \rightarrow 0}{\sim} \sum_{p} \hat{C}_{i j}^{p} \hat{C}_{k \ell}^{p} \hat{C}_{p p}^{1} e^{-\pi i S_{p}} b^{-2 \Delta_{p}} \cdot a^{2 \Delta_{p}-\Delta_{i}-\Delta_{j}-\Delta_{k}-\Delta_{\ell}} .
$$

The phase $e^{-\pi i S_{p}}=e^{-\pi i\left(h_{p}-\bar{h}_{p}\right)}$ in (2.9) is determined by our convention on how to continue the OPE (2.5) to the configuration in figure 2 where the result of the OPE of fields $\phi_{i}$ with $\phi_{j}$ and $\phi_{l}$ with $\phi_{k}$ results in fields at positions 0 and $i b$. We choose to continue the OPE so that the defect lines stay in the same topological arrangement and do not cross through the field insertion points which determines the phase uniquely.

In the $b \rightarrow 0$ limit we have to rearrange the $F$-defect before we are allowed to substitute the OPE. The relevant configuration is shown in figure $2 \mathrm{~b}$. We have

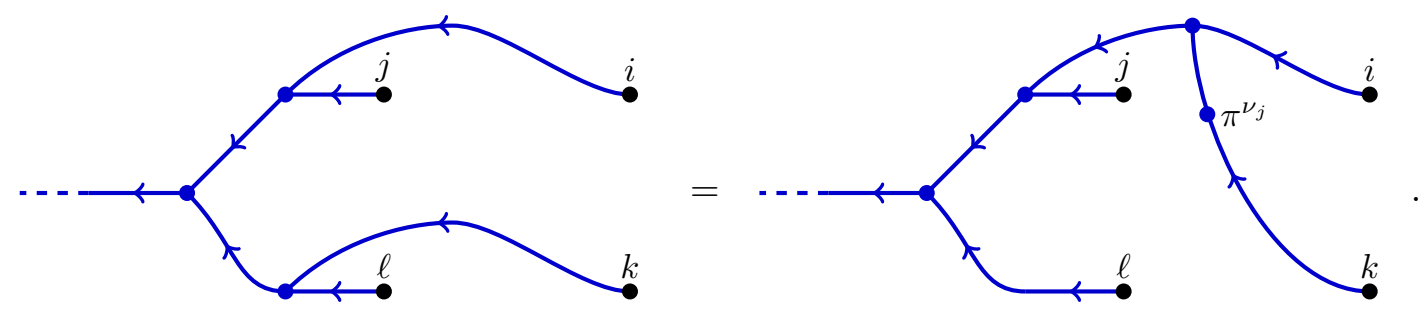


Here we used the associativity of the junction field and the effect of dragging an $F$-defect through a field, see figure 1e, 1f. The $\pi$-insertion contributes the sign factor $(-1)^{\nu_{j} \phi_{k}}$. The resulting graph of $F$-defects can be brought to the form in figure $2 \mathrm{~b}$ by using the associativity relation once more.

We see that bringing the spin structure to the form required for the OPE contributes a sign factor $(-1)^{\nu_{j} \phi_{k}}$. Another sign arises from parity as the order of $\phi_{j}$ and $\phi_{k}$ changes. Altogether we get

$f(a, b) \underset{b \rightarrow 0}{\sim}(-1)^{\left(\phi_{j}+\nu_{j}\right) \phi_{k}} \sum_{q} \hat{C}_{i k}^{q} \hat{C}_{j \ell}^{q} \hat{C}_{q q}^{1} e^{\frac{\pi i}{2}\left(2 S_{q}-S_{i}-S_{j}-S_{k}-S_{\ell}\right)} a^{-2 \Delta_{q}} \cdot b^{2 \Delta_{q}-\Delta_{i}-\Delta_{j}-\Delta_{k}-\Delta_{\ell}}$.

The rest of the computation is a standard manipulation of conformal blocks. The overall result is

$$
\hat{C}_{i k}^{q} \hat{C}_{j \ell}^{q} \hat{C}_{q q}^{1}=(-1)^{\left(\phi_{j}+\nu_{j}\right) \phi_{k}} \sum_{p} e^{\pi i\left(S_{i}+S_{\ell}-S_{p}-S_{q}\right)} F_{p q}\left[\begin{array}{cc}
j \ell \\
i k
\end{array}\right] F_{\bar{p} \bar{q}}\left[\begin{array}{l}
\bar{j} \\
\bar{\imath} \bar{\ell}
\end{array}\right] \hat{C}_{i j}^{p} \hat{C}_{k \ell}^{p} \hat{C}_{p p}^{1}
$$

Here, the entries " $i$ " and " $\vec{\imath}$ " in the two F-matrices refer to the holomorphic and antiholomorphic conformal weight $\left(h_{i}, \bar{h}_{i}\right)$ of $\phi_{i}$, etc.

\subsection{Symmetry properties of structure constants}

The four-point crossing relation determines how the structure constants behave under permutation of indices. Define

$$
\hat{C}_{i j k}=\hat{C}_{i j}^{k} \hat{C}_{k k}^{1}
$$

We obtain the following two relations by setting $\phi_{j}=1$ and $\phi_{\ell}=1$ in (2.12), respectively:

$$
\hat{C}_{k \ell i}=\hat{C}_{i k \ell}, \quad \hat{C}_{i j k}=(-1)^{\left(\phi_{j}+\nu_{j}\right) \phi_{k}} e^{\pi i\left(S_{k}+S_{j}-S_{i}\right)} \hat{C}_{i k j} .
$$

In particular, the $\hat{C}_{i j k}$ are cyclically symmetric. Using this we can rewrite the second equality above as a relation between $\hat{C}_{j k i}$ and $\hat{C}_{k j i}$. After relabelling and dividing by $\hat{C}_{k k}^{1}$ this implies

$$
\hat{C}_{j i}^{k}=(-1)^{\phi_{i}\left(\phi_{j}+\nu_{j}\right)} e^{\pi i\left(S_{i}+S_{j}-S_{k}\right)} \hat{C}_{i j}^{k} \stackrel{(*)}{=}(-1)^{\left(\phi_{i}+\nu_{i}\right) \phi_{j}} e^{\pi i\left(S_{k}-S_{i}-S_{j}\right)} \hat{C}_{i j}^{k} .
$$

In step $(*)$ we used that $e^{2 \pi i S_{i}}=(-1)^{\phi_{i}\left(\nu_{i}+1\right)}$, etc., from (2.3) and the fact that the OPE preserves the parity and spin gradings.

For the description of the classifying algebra below it will be important that structure constants involving only spinless fields are symmetric:

$$
\text { If } \quad S_{i}=S_{j}=S_{k}=0 \text { then } \hat{C}_{i j}^{k}=\hat{C}_{j i}^{k} .
$$

To see this, note that the only situation in which both expressions in (2.15) produce a sign is $\phi_{i}=\phi_{j}=1$ and $\nu_{i}=\nu_{j}=0$. But then (2.4) imposes $S_{i}, S_{j} \in \mathbb{Z}+\frac{1}{2}$, which we excluded. 


\subsection{Parity shift of the Ramond sector}

As we have seen in section 2.1 , the state space $\mathcal{H}_{F}$ of a fermionic CFT carries two $\mathbb{Z}_{2^{-}}$ gradings, namely even/odd and NS/R. Accordingly it splits into four direct summands,

$$
\begin{array}{lr}
\mathcal{H}_{F}^{\mathrm{NS}, \text { ev }} & \mathcal{H}_{F}^{\mathrm{R}, \mathrm{ev}} \\
\mathcal{H}_{F}^{\mathrm{NS}, \text { odd }} & \mathcal{H}_{F}^{\mathrm{R}, \text { odd }}
\end{array}
$$

As before, for a primary bulk field $\phi_{i}$, its parity is $\left|\phi_{i}\right| \in\{0,1\}$ (with 0 being even) and its spin grade is $\nu_{i} \in\{0,1\}$ (with 0 being NS). Let $\hat{C}_{i j}^{k}$ be a solution of the bulk crossing relation (2.12).

Given such a solution, we can construct a new fermionic CFT as follows. The new state space $\widetilde{\mathcal{H}}_{F}$ agrees with the old one, except for a parity shift in the Ramond sector:

$$
\begin{aligned}
\widetilde{\mathcal{H}}_{F}^{\mathrm{NS}, \text { ev }} & :=\mathcal{H}_{F}^{\mathrm{NS}, \text { ev }} & \widetilde{\mathcal{H}}_{F}^{\mathrm{R}, \mathrm{ev}} & :=\mathcal{H}_{F}^{\mathrm{R}, \text { odd }} \\
\widetilde{\mathcal{H}}_{F}^{\mathrm{NS}, \text { odd }} & :=\mathcal{H}_{F}^{\mathrm{NS}, \text { odd }} & \widetilde{\mathcal{H}}_{F}^{\mathrm{R}, \text { odd }} & :=\mathcal{H}_{F}^{\mathrm{R}, \text { ev }}
\end{aligned}
$$

That is, the old and new gradings are related by

$$
\left|\widetilde{\phi}_{i}\right|=\left|\phi_{i}\right|+\nu_{i}, \quad \widetilde{\nu}_{i}=\nu_{i} .
$$

The new structure constants are related to the old ones by signs,

$$
\widetilde{C}_{i j}^{k}:=(-1)^{\nu_{i} \phi_{j}} \hat{C}_{i j}^{k} .
$$

It is a straightforward computation to see that $\widetilde{C}_{i j}^{k}$ again solves the crossing relation (2.12) (and hence also has the symmetry properties stated in section 2.4).

Applying the shift operation twice produces the structure constants $(-1)^{\nu_{i} \nu_{j}} \hat{C}_{i j}{ }^{k}$. The extra sign can be absorbed into the normalisation of the fields (e.g. multiply all Ramond fields by $i$ ), so that this reproduces the theory one started from. ${ }^{2}$

The parity shifted theory may or may not be isomorphic to the unshifted theory. For example, parity shifting the fermionic Ising model produces an equivalent theory, while parity shifting the fermionic minimal model $\operatorname{FM}(3,8)$ produces an inequivalent theory, see sections 5.1 and 6.2

\section{Classifying algebras}

In this section we use the bulk structure constants of a fermionic CFT to define several types of fermionic classifying algebras. These are semisimple super-algebras, graded by parity, whose direct summands are in 1-1 correspondence to boundary conditions (section 3.1), defects, or interfaces (section 3.2), depending on the algebra under consideration. In section 3.3 we observe that either one of these classifying algebras in a fermionic CFT is isomorphic to the corresponding algebra in the parity shifted CFT as an ungraded algebra, but typically not as a super-algebra.

\footnotetext{
${ }^{2}$ It is possible to modify (2.20) so that applying the parity shift twice gives back precisely the structure constants one started from. For example, with $\gamma_{a}=e^{-\pi i\left(S_{a}+\phi_{a} / 2\right)}$ one can set $\widetilde{C}_{i j}^{k}=(-1)^{\nu_{i} \phi_{j}} \gamma_{i} \gamma_{j} / \gamma_{k} \hat{C}_{i j}^{k}=$ $(-1)^{\left(\nu_{i}+\phi_{i}\right) \phi_{j}} e^{\pi i\left(S_{k}-S_{i}-S_{j}\right)} \hat{C}_{i j}^{k}=\hat{C}_{j i}^{k}$, where in the last step we used (2.15).
} 


\subsection{Bulk-boundary OPE and boundary classifying algebra}

\subsubsection{Conventions for bulk-boundary OPE and boundary OPE}

We now consider the theory on the upper half plane with some conformally invariant boundary condition $\alpha$ placed on the real line. As for bulk fields, boundary fields will also serve as starting point for an $F$-defect. We denote the space of boundary fields by $\mathcal{H}_{F}^{(\alpha)}$. It is again $\mathbb{Z}_{2}$-graded by parity, ${ }^{3}$

$$
\mathcal{H}_{F}^{(\alpha)}=\mathcal{H}_{F}^{(\alpha), \text { ev }} \oplus \mathcal{H}_{F}^{(\alpha), \text { odd }} .
$$

Our convention for the defect arrangement near an insertion of a boundary field $\psi \in \mathcal{H}_{F}^{(\alpha)}$ is:

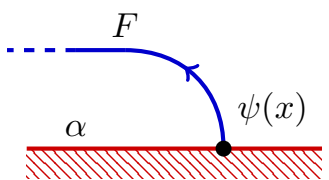

The bulk-boundary structure constants $B_{\psi}^{\phi}$ are defined as, for a primary bulk field $\phi \in \mathcal{H}_{F}$,

$$
\phi(x+i y)=\sum_{\psi} B_{\psi}^{\phi}(2 y)^{h_{\psi}-\Delta_{\phi}} \psi(x)+(\text { desc. })
$$

where the sum runs over a basis of primary fields $\psi$ in $\mathcal{H}_{F}^{(\alpha)}$. In pictures the above relation looks as follows,

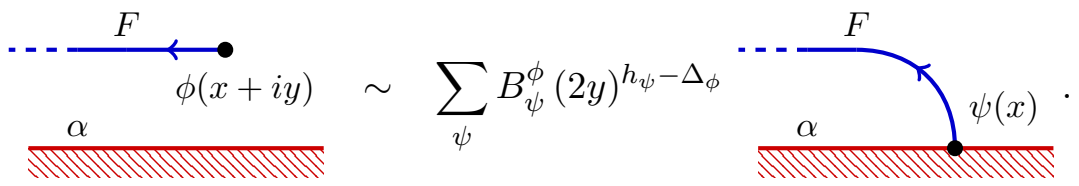

We will also use the OPE of boundary fields, for which our convention is, for $x>y$,

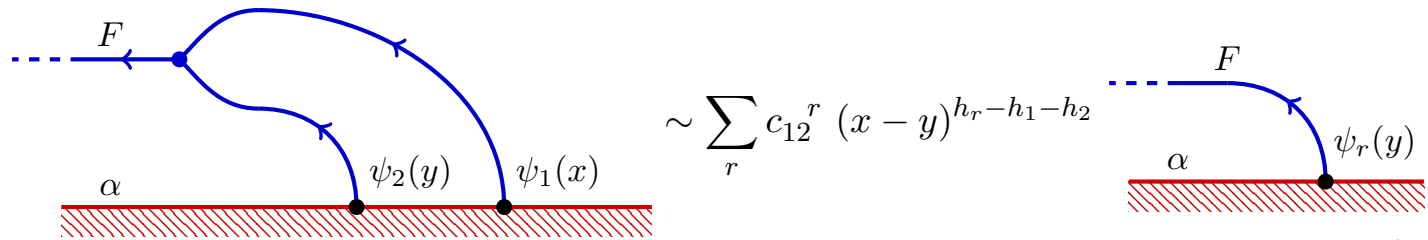

where the sum runs over a basis of primary fields $\psi_{r}$ in $\mathcal{H}_{F}^{(\alpha)}$.

We will only consider boundary conditions for which the bulk-boundary OPE and the boundary OPE preserve parity.

\subsubsection{Bulk-boundary crossing relation}

Let $g(a, b)$ stand for the correlator of two bulk fields and one boundary field shown in figure 3. The crossing relation is obtained by comparing the $a \rightarrow 0$ and $b \rightarrow 0$ limit.

\footnotetext{
${ }^{3}$ If there are holomorphic and antiholomorphic fermions, one can define a monodromy for fermion fields around boundary (changing) fields. In this sense the space of boundary fields may also split into NS- and R-sectors. But this split does not have a geometric counterpart in the present setting: we consider a surface with a single spin structure, and near a boundary point a spin structure is unique up to isomorphism.
} 


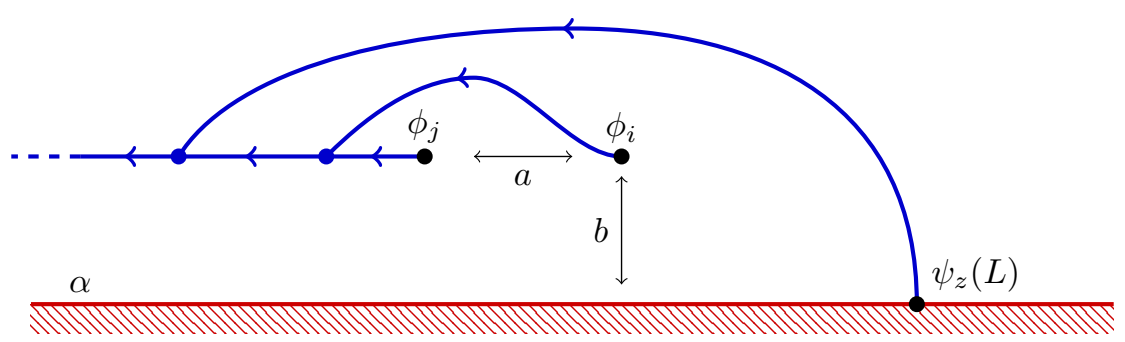

Figure 3. Two bulk fields and one boundary field together with their defect lines as used in computing the crossing constraint linking bulk-boundary couplings and bulk structure constants. In the correlator $g(a, b)$, the boundary field $\psi_{z}$ inserted at $L$ is moved off to $\infty$.

It turns out that this relation does not involve parity signs or spin structure signs. The computation is thus standard and we will be very brief. The asymptotic behaviour of $g(a, b)$ in the two limits is

$$
\begin{aligned}
& g(a, b) \underset{a \rightarrow 0}{\sim} \sum_{k} \hat{C}_{i j}^{k} B_{z}^{k} c_{z z}^{1}(2 b)^{h_{z}-\Delta_{k}} \cdot a^{\Delta_{k}-\Delta_{i}-\Delta_{j}}, \\
& g(a, b) \underset{b \rightarrow 0}{\sim} \sum_{x, y} B_{x}^{i} B_{y}^{j} c_{x y}^{z} c_{z z}^{1} a^{h_{z}-h_{x}-h_{y}} \cdot(2 b)^{h_{x}+h_{y}-\Delta_{i}-\Delta_{j}} .
\end{aligned}
$$

The crossing relation now follows from a computation with five-point Virasoro conformal blocks similar to those in [16], and the result is

$$
\begin{aligned}
B_{x}^{i} B_{y}^{j} c_{x y}^{z}=\sum_{k, r} \hat{C}_{i j}^{k} B_{z}^{k} e^{\frac{i \pi}{2}\left(S_{k}-S_{i}-S_{j}\right)} e^{\frac{i \pi}{2}\left(h_{x}-h_{y}-h_{z}\right)} & \\
& \times e^{i \pi\left(h_{r}-\bar{h}_{i}\right)} F_{k r}\left[\begin{array}{cc}
\bar{k} & j \\
z & i
\end{array}\right] F_{\bar{k}, y}\left[\begin{array}{ll}
j & \bar{j} \\
r & \bar{l}
\end{array}\right] F_{r x}\left[\begin{array}{ll}
i & \bar{z} \\
z & y
\end{array}\right] .
\end{aligned}
$$

If the boundary field $\psi_{z}$ has weight zero (but could still be of either parity, see next subsection), this relation simplifies to

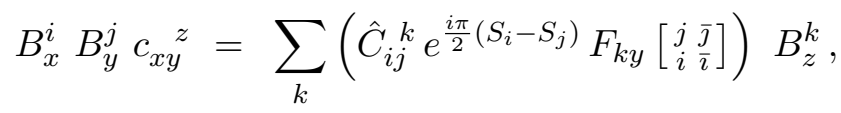

where we used that $B_{z}^{k} \neq 0$ requires $h_{k}=\bar{h}_{k}$. If the boundary fields $\psi_{x}, \psi_{y}, \psi_{z}$ all have weight zero (but could again be of either parity), this relation simplifies further to

$$
B_{x}^{i} B_{y}^{j} c_{x y}^{z}=\sum_{k}\left(\hat{C}_{i j}^{k} F_{k 0}\left[\begin{array}{ll}
j & j \\
i & i
\end{array}\right]\right) B_{z}^{k}
$$

The corresponding relation for bosonic theories was derived in [17].

\subsubsection{Boundary classifying algebra: bosonic case}

Let us first recall the situation when there are just bosonic (i.e. parity even) fields and consider the space of weight zero boundary fields which can arise in the bulk-boundary OPE. Looking at the right hand side of the crossing constraint (3.9), this is symmetric under $i \leftrightarrow j$ and as a consequence $c_{x y}^{z}$ must be symmetric under $x \leftrightarrow y$. This means that 
the weight zero boundary fields which arise in the bulk-boundary OPE form a commutative, associative algebra. If one restricts one's attention to situations where this algebra is semisimple, by the Wedderburn theorem it must be equal to a direct sum of copies of $\mathbb{C}$, each of which can be considered as the identity operator on an elementary boundary condition.

Due to this observation, it is enough to consider elementary boundary conditions, i.e. boundary conditions on which there is a single boundary field of weight 0 (the identity field). The bosonic version of (3.9) reduces to

$$
B^{i} B^{j}=\sum_{k}\left(C_{i j}^{k} F_{k 0}\left[\begin{array}{cc}
j & j \\
i & i
\end{array}\right]\right) B^{k} .
$$

This equation can be simply seen as a set of equations for the constants $B^{i}$, but as shown in $[3,18]$ this is also the defining relation for a commutative associative algebra with generators $B^{k}$, the (bosonic) boundary classifying algebra. From this point of view, the elementary boundary conditions correspond to the one-dimensional representations of this algebra, or, equivalently, to the number of summands $\mathbb{C}$ in the direct sum decomposition of $(3.10)$.

\subsubsection{Boundary classifying algebra: fermionic case}

In the fermionic case, we equally take the coefficients $\hat{C}_{i j}^{k} F_{k 0}\left[\begin{array}{l}j \\ i\end{array}\right]$ constants of an algebra with generators $B^{i}$,

$$
B^{i} B^{j}=\sum_{k}\left(\hat{C}_{i j}^{k} F_{k 0}\left[\begin{array}{cc}
j & j \\
i & i
\end{array}\right]\right) B^{k},
$$

where the indices $i, j, k$ now run over all spinless fields of either parity. We denote this algebra by $\hat{\mathcal{B}}$ and call it the fermionic boundary classifying algebra. In the examples we consider in sections 5 and 6 , this is indeed an associative algebra which is in addition semisimple. In [2] we will show that (3.11) defines a semisimple associative algebra for fermionic rational CFTs in general.

While the $\hat{C}_{i j}{ }^{k}$ are not necessarily symmetric under $i \leftrightarrow j$, we saw in (2.16) that they are symmetric for all $i, j$ and $k$ which have spin 0 and which can therefore couple to boundary fields of weight 0 . It follows that the algebra $\hat{\mathcal{B}}$ is commutative, $B^{i} B^{j}=B^{j} B^{i}$.

Since the bulk fields are graded by their parity, we can also view $\hat{\mathcal{B}}$ as a super-algebra with the parity of the generator $B^{i}$ being the same as that of the field $\phi_{i}$. As the above commutativity relation does not involve parity signs, $\hat{\mathcal{B}}$ is in general not super-commutative. Note that $\hat{\mathcal{B}}$ is in fact bi-graded, by parity and by spin, since the bulk structure constants $\hat{C}_{i j}{ }^{k}$ preserve both these gradings.

Let us assume that $\hat{\mathcal{B}}$ is semisimple. Since $\hat{\mathcal{B}}$ is commutative (rather than supercommutative), the super-Wedderburn theorem [19, corollary 2.12] states that the algebra splits into a sum of copies of $\mathbb{C}^{1 \mid 0}=\mathbb{C}$ and the Clifford algebra $Q(2) \equiv C \ell_{1}$ which is twodimensional with one odd generator, $a$, satisfying $a^{2}=1$. In other words, $\hat{\mathcal{B}}$ is equal to a sum of $m$ copies of $\mathbb{C}$ with generators $e_{\alpha}$ (where $\alpha$ labels the copy) and $n$ copies of $C \ell_{1}$ 
with even generator $f_{\beta}$ and odd generator $a_{\beta}$,

$$
\hat{\mathcal{B}}=\left(\bigoplus^{m} \mathbb{C} e_{\alpha}\right) \oplus\left(\bigoplus^{n}\left(\mathbb{C} f_{\beta} \oplus \mathbb{C} a_{\beta}\right)\right)
$$

These generators satisfy the relations

$$
e_{\alpha}^{2}=e_{\alpha}, \quad f_{\beta}^{2}=f_{\beta}, \quad f_{\beta} a_{\beta}=a_{\beta}, \quad a_{\beta}^{2}=f_{\beta}, \quad x_{\gamma} y_{\delta}=0, \gamma \neq \delta .
$$

After analysing $\hat{\mathcal{B}}$ in some detail, let us return to the bulk-boundary crossing constraint (3.9). Analogous to the bosonic case, we conclude that the weight zero boundary fields which arise in the bulk-boundary OPE form an associative super-algebra which is commutative but not necessarily super-commutative, and which is in addition semisimple. ${ }^{4}$ Again by the super-Wedderburn theorem, this algebra of boundary fields decomposes into a direct sum of $\mathbb{C}$ 's and $C \ell_{1}$ 's. We will call a boundary condition of the fermionic CFT elementary if its weight zero boundary fields consist of either exactly $\mathbb{C}$ or exactly $C \ell_{1}$. Note that in the standard basis of $\mathbb{C}$ and $C \ell_{1}$, the boundary structure constants $c_{x y}^{z}$ in (3.9) are either 0 or 1 .

In the case of $C \ell_{1}$ there is a weight zero boundary fermion $a$ which satisfies $a^{2}=1$. Such weight-zero fermions are well-known and appear in treatments of the boundary Ising model, for example in [5-8].

We note that as an ungraded algebra, $C \ell_{1}$ is isomorphic to $\mathbb{C} \oplus \mathbb{C}$, but the corresponding generators would not have a fixed parity. Consequently, it is not possible to split a boundary condition with weight zero field content $C \ell_{1}$ into two more elementary boundaries without breaking parity-preservation of the bulk-boundary OPE.

We conclude that the indices $\alpha$ in (3.12) label the different elementary boundary conditions: Each pair $\left\{f_{\beta}, a_{\beta}\right\}$ corresponds to a boundary condition which supports an odd weight 0 field, while each generator $e_{\alpha}$ corresponds to a boundary condition which does not. The bulk-boundary structure constants are given by the action of the algebra elements $B^{i}$ on the generators,

$$
B^{i} e_{\alpha}=B_{1}^{(\alpha) i} e_{\alpha}, \quad B^{i} f_{\beta}=B_{1}^{(\beta) i} f_{\beta}+B_{a}^{(\beta) i} a_{\beta}, \quad B^{i} a_{\beta}=B_{1}^{(\beta) i} a_{\beta}+B_{a}^{(\beta) i} f_{\beta} .
$$

Here, we added the superscripts $(\alpha),(\beta)$ to distinguish different solutions to (3.9).

Analogous to the bosonic case, there is a relation between elementary boundary conditions and representations of the fermionic boundary classifying algebra. Namely,

\{elementary boundary conditions $\}$

$$
\begin{aligned}
& \cong\left\{\text { irreducible } \mathbb{Z}_{2} \text {-graded } \hat{\mathcal{B}} \text {-modules that are submodules of } \hat{\mathcal{B}}\right\} \\
& \cong\left\{\text { irreducible } \mathbb{Z}_{2} \text {-graded } \hat{\mathcal{B}} \text {-modules up to even or odd isomorphisms }\right\} \text {. }
\end{aligned}
$$

Both equivalences follow from the description of elementary boundary conditions in terms of direct summands in (3.12). Note, however, that elementary boundary conditions do not

\footnotetext{
${ }^{4}$ Semisimplicity follows from that of $\hat{\mathcal{B}}$. Indeed, the coefficients $B_{x}^{i}$ in (3.9) can be understood as the coefficients of a homomorphism of super-algebras from $\hat{\mathcal{B}}$ to the algebra of weight zero boundary fields. Since $\hat{\mathcal{B}}$ is by assumption semisimple, so is its image under a homomorphism.
} 
correspond to irreducible $\mathbb{Z}_{2}$-graded $\hat{\mathcal{B}}$-modules up to only even isomorphism (this would count summands $\mathbb{C}$ with a factor of two and summands $C \ell_{1}$ with a factor of one). ${ }^{5}$

As can be seen from the structure of the boundary classifying algebra of fermionic theories, restricting to the bosonic generators and removing the fermionic generators reduces each copy of $C \ell_{1}=\mathbb{C}^{1 \mid 1}$ to just $\mathbb{C}$. In this way we see that the elementary boundary conditions of a fermionic theory are in $1-1$ correspondence with the elementary boundary conditions of its bosonic projection (the related "spin theory"); consideration of the fermionic generators allows one to see which boundary conditions support a fermionic weight zero field and are hence "supersymmetric" as we discuss below.

\subsection{Bulk-defect OPE and defect classifying algebra}

Topological defects in a conformal field theory $\mathcal{C}$ have been long known to be equivalent to a particular class of conformal boundary conditions (a special case of so-called permutation boundary conditions [20]) on the doubled model $\mathcal{C} \times \overline{\mathcal{C}}$, and so can be studied from that perspective. It can, however, also be useful to consider them in their own right and that is what we do here.

A topological defect will always have a non-trivial set of defect fields, as it will always support the full set of bulk fields; in the case of the trivial identity defect this is exactly the space of defect fields, but in general it will be larger.

It does not add any difficulty to generalise the situation slightly to topological interfaces with a CFT $\mathcal{C}$ above the interface and a CFT $\mathcal{C}^{\prime}$ below the interface. Below we will sometimes use the term defect to include both situations, with $\mathcal{C}$ and $\mathcal{C}^{\prime}$ being equal or different.

The sewing constraint for topological defects is almost identical to the bulk crossing symmetry constraint (2.12), with the replacement of some bulk structure constants by bulk-defect structure constants and defect-defect structure constants. We shall here focus only on weight zero fields on the defect as this is sufficient to derive the defect classifying algebra. The bulk-defect structure constants in the OPE of two bulk fields $\phi_{i}$ (above the defect) and $\phi_{k}^{\prime}$ (below the defect) to a weight zero defect field $\vartheta_{z}$ will be denoted by $D_{z}^{i k}$ and the OPE of weight-zero defect fields will be taken to have structure constants $c_{x y}{ }^{z}$ :

$$
\begin{aligned}
\phi_{i}(a+i b) \phi_{k}^{\prime}(a-i b) & =\delta_{h_{i}, h_{k}} \delta_{\bar{h}_{i}, \bar{h}_{k}} \frac{D_{z}^{i k}}{|b|^{2 \Delta_{i}}} \vartheta_{z}(a)+\ldots, \\
\vartheta_{x} \vartheta_{y} & =c_{x y}{ }^{z} \vartheta_{z} .
\end{aligned}
$$

The result is the defect sewing constraint

$$
D_{x}^{i k} D_{y}^{j \ell} c_{x y}^{z}=(-1)^{\left(\phi_{j}+\nu_{j}\right) \phi_{k}} \sum_{p, q} e^{\pi i\left(S_{i}+S_{\ell}-S_{p}\right)} F_{p 0}\left[\begin{array}{ll}
j & \ell \\
i & k
\end{array}\right] F_{\bar{p} 0}\left[\begin{array}{l}
\bar{j} \\
\bar{\imath} \bar{\ell}
\end{array}\right] \hat{C}_{i j}^{p} \hat{C}_{k \ell}^{\prime q} D_{z}^{p q} .
$$

\footnotetext{
${ }^{5}$ Nonetheless, we will see in [2] that it is in fact natural to say that each elementary boundary condition comes in two varieties, which differ by an overall sign in the boundary state (this then counts each elementary boundary condition with a factor of two).
} 
As in the boundary case, we can consider the couplings to the weight zero fields as generators $D^{i k}$ of an algebra $\hat{\mathcal{D}}$ - the fermionic defect classifying algebra - defined in terms of the constants on the right hand side of (3.17),

$$
D^{i k} D^{j \ell}=(-1)^{\left(\phi_{j}+\nu_{j}\right) \phi_{k}} \sum_{p, q} e^{\pi i\left(S_{i}+S_{\ell}-S_{p}\right)} F_{p 0}\left[\begin{array}{ll}
j & \ell \\
i & k
\end{array}\right] F_{\bar{p} 0}\left[\begin{array}{l}
\bar{j} \bar{\ell} \\
\bar{\imath} \bar{k}
\end{array}\right] \hat{C}_{i j}^{p} \hat{C}_{k \ell}^{\prime q} D^{p q} .
$$

Here, the pairs $i k, j \ell$ and $p q$ have to satisfy the condition that for each pair $x y$ the bulk fields $\phi_{x}$ and $\phi_{y}^{\prime}$ have the same left conformal weight and the same right conformal weight. This implies, for example, that $F_{p 0}\left[\begin{array}{ll}j & \ell \\ i & k\end{array}\right]=F_{p 0}\left[\begin{array}{ll}j & j \\ i & i\end{array}\right]$.

The algebra $\hat{\mathcal{D}}$ is commutative and associative (at least in fermionic rational CFTs [2]). We take the generators $D^{i j}$ to inherit the parity of the product of fields $\phi_{i} \phi_{j}^{\prime}$, so that $\hat{\mathcal{D}}$ is also a super-algebra (not necessarily super-commutative). As in the boundary situation, we assume that $\hat{\mathcal{D}}$ is semisimple, in which case it decomposes into a direct sum of copies of $\mathbb{C}$ and $C \ell_{1}$ which are in 1-1 correspondence with the solutions of the defect sewing constraints and hence in correspondence with the defect operators (up to an overall sign) and elementary defect conditions.

In the case $\mathcal{C}=\mathcal{C}^{\prime}$, one generic solution for the $D_{z}^{i k}$ is provided by the trivial defect. The only weight zero field on the trivial defect is the identity bulk field 1 , and we can set $D_{1}^{i k}=\hat{C}_{i k}^{1}$. The bulk-defect crossing relation (3.17) then turns into a special case of the bulk crossing relation (2.12).

Interfaces between fermionic and bosonic theories can also be treated by the above classifying algebra by simply choosing one of the two CFTs to be purely even.

In the purely bosonic case, classifying algebras for topological defects were studied in [4].

As opposed to case of boundary conditions, the number of defects of a fermionic theory is larger than the number of defects of its bosonic projection since there is a bosonic generator $D^{\phi \phi}$ of $\mathcal{D}$ for each field $\phi$, whether it is bosonic or fermionic. Each defect of the bosonic theory can be associated with one or more defects in the fermionic theory (as it happens, in the examples we have looked at, each bosonic defect is associated to two defects in the fermionic theory).

\subsection{Classifying algebras in the parity shifted theory}

When comparing the various classifying algebras between a fermionic theory and its parityshifted version, one finds that they are isomorphic as ungraded algebras, and that one can give an isomorphism by a simple rescaling of the generators.

However, we stress that the fermionic classifying algebras will in general not be isomorphic as super-algebras (with $\mathbb{Z}_{2}$-grading given by parity). We will see this explicitly in the example of $F M(3,8)$ in section 6.2 .

\subsubsection{Fermionic boundary classifying algebra}

Let us write $B^{i}$ for the generators in the unshifted theory as in section 3.1.4 and denote the structure constants of the fermionic boundary classifying algebra by $\beta_{i j}{ }^{k}$, such that (3.11) becomes $B^{i} B^{j}=\sum_{k} \beta_{i j}^{k} B^{k}$. The bulk structure constants in the parity-shifted theory 
are given in (2.20). If we denote the generators and structure constants of the classifying algebra of the parity shifted theory by $\widetilde{B}^{i}$ and $\widetilde{\beta}_{i j}^{k}$, we get

$$
\widetilde{B}^{i} \widetilde{B}^{j}=\sum_{k} \widetilde{\beta}_{i j}^{k} \widetilde{B}^{k} \quad \text { with } \quad \widetilde{\beta}_{i j}^{k}=(-1)^{\nu_{i} \phi_{j}} \beta_{i j}^{k}
$$

In general, the factor $(-1)^{\nu_{i} \phi_{j}}$ cannot be absorbed into a rescaling, ${ }^{6}$ but since certain of the $\beta_{i j}{ }^{k}$ are zero, here this will be possible. Explicitly, we may identify $\widetilde{B}^{i}=\lambda_{i} B^{i}$ to get

$$
\widetilde{\beta}_{i j}^{k}=\frac{\lambda_{i} \lambda_{j}}{\lambda_{k}} \beta_{i j}^{k} \quad \text { with } \quad \lambda_{x}=e^{\frac{\pi i}{2} \phi_{x}}
$$

The scalar coefficients $\lambda$ are not unique, and we just exhibit one possible solution. To verify the above equality, first note that $\lambda_{i} \lambda_{j} / \lambda_{k}=(-1)^{\phi_{i} \phi_{j}}$. One thus needs to check that $(-1)^{\nu_{i} \phi_{j}}=(-1)^{\phi_{i} \phi_{j}}$ whenever $\beta_{i j}^{k} \neq 0$. Since all NS-sector generators in the boundary classifying algebra are parity-even, we only need to consider the case that $B^{i}$ and $B^{j}$ are from the R-sector, and that $B^{i}$ is even and $B^{j}$ is odd. But then $\beta_{i j}^{k}=0$, as $B^{k}$ would then need to be odd and in the NS-sector.

\subsubsection{Fermionic defect classifying algebra}

Let us use the letter " $U$ " for the unshifted theory and " $P$ " for the parity shifted theory. Here we will give the relation between the defect classifying algebras of types $U-U, U-P$, $P-U$ and $P-P$, where the first letter refers to the theory in the upper half plane. The $U-U$-case is as given in section 3.2 and will be our reference case:

$$
D^{i k} D^{j \ell}=\sum_{p, q} \beta_{i k, j \ell}^{(U U) p q} D^{p q}
$$

with $\beta_{i k, j \ell}^{(U U) p q}$ determined by (3.18). A computation similar to the boundary classifying algebra gives (all parities are stated with respect to the unshifted theory)

$$
\begin{aligned}
& \beta_{i k, j \ell}^{(U P) p q}=(-1)^{\nu_{k}\left(\phi_{j}+\phi_{\ell}+\nu_{\ell}\right)} \beta_{i k, j \ell}^{(U U) p q}=\frac{\lambda_{i k} \lambda_{j \ell}}{\lambda_{p q}} \beta_{i k, j \ell}^{(U U) p q}, \quad \lambda_{x y}=e^{\frac{\pi i}{2}\left(\phi_{x}+\phi_{y}-2 \phi_{x} \phi_{y}+\nu_{y}\right)}, \\
& \beta_{i k, j \ell}^{(P U) p q}=(-1)^{\nu_{i} \phi_{j}+\nu_{\ell} \phi_{k}} \beta_{i k, j \ell}^{(U U) p q}=\frac{\lambda_{i k}^{\prime} \lambda_{j \ell}^{\prime}}{\lambda_{p q}^{\prime}} \beta_{i k, j \ell}^{(U U) p q}, \quad \lambda_{x y}^{\prime}=e^{\frac{\pi i}{2}\left(\phi_{x}+\phi_{y}-2 \phi_{x} \phi_{y}\right)} e^{2 \pi i S_{y}}, \\
& \beta_{i k, j \ell}^{(P P) p q}=(-1)^{\nu_{\ell} \phi_{k}+\nu_{k} \phi_{\ell}} \beta_{i k, j \ell}^{(U U) p q}=\frac{\lambda_{i k}^{\prime \prime} \lambda_{j \ell}^{\prime \prime}}{\lambda_{p q}^{\prime \prime}} \beta_{i k, j \ell}^{(U U) p q},
\end{aligned}
$$

To verify these equations one needs to use that for a generator $D^{x y}$ one always has $\nu_{x}=\nu_{y}$ (but not necessarily $\phi_{x}=\phi_{y}$ ) as otherwise the bulk-defect OPE does not contain 1 or $a$.

\footnotetext{
${ }^{6}$ The reason for this is that $c_{i, j}=(-1)^{\nu_{i}} \phi_{j}$ is a non-exact two-cocycle on $\mathbb{Z}_{2} \times \mathbb{Z}_{2}$ as witnessed by the fact that it is not symmetric in $i, j$.
} 


\begin{tabular}{|c|c|c|c|c|c|c|}
\hline$h_{G}$ & $\frac{1}{2}$ & $\frac{3}{2}$ & $\frac{5}{2}$ & $\frac{7}{2}$ & $\frac{9}{2}$ & $\cdots$ \\
\hline$(p, q)$ & $(3,4)$ & $(3,8),(4,5)$ & $(4,7)$ & $(3,16),(4,9)$ & $(3,20),(4,11),(5,8)$ & $\ldots$ \\
\hline$c(p, q)$ & $\frac{1}{2}$ & $-\frac{21}{4}, \frac{7}{10}$ & $-\frac{13}{14}$ & $-\frac{161}{8},-\frac{19}{6}$ & $-\frac{279}{10},-\frac{125}{22},-\frac{7}{20}$ & $\cdots$ \\
\hline
\end{tabular}

Table 1. Virasoro minimal models admitting a fermionic generator $G$ listed by increasing $h_{G}$.

\section{Fermionic Virasoro minimal models}

The examples we shall consider are all Virasoro minimal, i.e. the Hilbert space is formed from a finite set of Virasoro representations. This might seem odd, as it might be more natural to start with a super-algebra for which there is naturally a fermionic interpretation, but as we shall see it provides ample examples, not only infinite series which extend the bosonic Virasoro minimal models but also the simplest example, a free fermion, as well as examples with super-Virasoro symmetry and extended (W-algebra type) fermionic algebras.

In this section we give the bulk structure constants for fermionic A- and D-type minimal models, in section 5 we treat the free fermion in detail, and in section 6 we give further Virasoro examples.

Consider the Virasoro algebra at the minimal model central charge $c(p, q)=1-6(p-$ $q)^{2} /(p q)$ with $p, q$ coprime integers greater than 1 . We recall that the possible Virasoro representations are labelled by two integers $(r, s)$ where $1 \leq r<p$ and $1 \leq s<q$ with the identification $(r, s) \simeq(p-r, q-s)$; for more details see [21].

The fermionic generator $G$ we consider has Kac-labels $(1, q-1) \sim(p-1,1)$ and is the unique non-trivial simple current at that central charge. Note that for $G$ to indeed be different from the vacuum representation we actually need $p, q>2$. The conformal weight of $G$ is $h_{G}=\frac{1}{4}(p-2)(q-2)$. The condition that $h_{G} \in \mathbb{Z}+\frac{1}{2}$ amounts to

$$
p=2 n+1, q=4 k, \quad \text { or equivalently } p=4 k, q=2 n+1,
$$

$k, n \geq 1$, in which case $h_{G}=\frac{1}{2}(2 n-1)(2 k-1)$. The first few values of $h_{G}$ are realised in the models in table 1 . The next value of $h_{G}$ realised in a unitary model is $h_{G}=\frac{15}{2}$ at central charge $c(7,8)=\frac{25}{28}$.

Recall that the possible different bosonic field theories with this central charge are labelled by a pair of simply-laced Lie algebras with Coxeter numbers $p, q[9]$. We will give two explicit solutions to the fermionic crossing relation (2.12). One is an extension of the minimal model of type $M\left(A_{p-1}, A_{q-1}\right)$ which we will call the fermionic Virasoro minimal model $F M\left(A_{p-1}, A_{q-1}\right)$ or $F M(p, q)$ for short. The other is an extension of the minimal model $M\left(A_{p-1}, D_{q / 2+1}\right)$ which we will call $F M\left(A_{p-1}, D_{q / 2+1}\right)$ or $\widetilde{F M}(p, q)$ for short. By an extension we mean that the fermionic model contains the full bosonic field theory as a sub-theory. The two models $F M(p, q)$ and $\widetilde{F M}(p, q)$ are obtained from each other by shifting the Ramond sector parity as in section 2.5. 


\subsection{A-type fermionic models}

Let $\mathcal{I}$ be an indexing set for the Kac-table modulo its $\mathbb{Z}_{2}$-identification $(r, s) \sim(p-r, q-s)$, and let $M_{a}, a \in \mathcal{I}$ be the corresponding irreducible Virasoro representation. In the setting of [1, section 5], the defect $F$ relevant for $A$-type fermionic models is the superposition of the identity defect and a parity shifted version of the topological defect labelled by the representation $G=M_{(1, q-1)}$. (What we call $F$ here is called $A$ in [1].) The splitting into NS- and R-sector depends on the sign of the ratio of $S$-matrices $S_{G, a} / S_{G, 0} \in\{ \pm 1\}$, with +1 being the NS-sector and -1 the R-sector. Explicitly, for $a=(r, s)$ we have $S_{G, a} / S_{G, 0}=(-1)^{q r+p s+1}$ so that the set $\mathcal{I}$ splits as

$$
\mathcal{I}_{\mathrm{NS}}=\{(r, s) \in \mathcal{I} \mid q r+p s \text { odd }\}, \quad \mathcal{I}_{\mathrm{R}}=\{(r, s) \in \mathcal{I} \mid q r+p s \text { even }\} .
$$

The state space $\mathcal{H}_{F}$ of $F M\left(A_{p-1}, A_{q-1}\right)$ splits into the four sectors in (2.17) as follows:

$$
\begin{array}{rlrl}
\mathcal{H}_{F}^{\mathrm{NS}, \mathrm{ev}} & :=\bigoplus_{a \in \mathcal{I}_{\mathrm{NS}}} M_{a} \otimes \bar{M}_{a}, & \mathcal{H}_{F}^{\mathrm{R}, \mathrm{ev}} & :=\bigoplus_{a \in \mathcal{I}_{\mathrm{R}}} M_{a} \otimes \bar{M}_{a}, \\
\mathcal{H}_{F}^{\mathrm{NS}, \mathrm{odd}} & :=\bigoplus_{a \in \mathcal{I}_{\mathrm{NS}}} M_{a} \otimes \bar{M}_{G a}, & \mathcal{H}_{F}^{\mathrm{R}, \mathrm{odd}}:=\bigoplus_{a \in \mathcal{I}_{\mathrm{R}}} M_{a} \otimes \bar{M}_{G a},
\end{array}
$$

where $G a$ is the result of the fusion product of $G$ and $a$. That is, for $a=(r, s)$ we have $G a=(r, q-s) \sim(p-r, s)$. Note that $\mathcal{H}_{F}^{\mathrm{NS}, \text { odd }}$ contains a holomorphic field of weight $\left(h_{G}, 0\right)$ and an anti-holomorphic one of weight $\left(0, h_{G}\right)$.

We will use the notation $\phi_{a}^{e}$ for the primary field in $\mathcal{H}_{F}^{\text {ev }}$ of conformal weights $\left(h_{a}, h_{a}\right)$, and $\phi_{a}^{o}$ for the primary field in $\mathcal{H}_{F}^{\text {odd }}$ of conformal weights $\left(h_{a}, h_{G a}\right)$. This notation is slightly asymmetric in that $\phi_{G}^{o}$ is the field of weight $\left(h_{G}, 0\right)$ while $\phi_{1}^{o}$ is that of weight $\left(0, h_{G}\right)$. Suppose we take $q=4 k$ in (4.1). Then $G a=a$ if and only if $a=\left(r, \frac{q}{2}\right)=(r, 2 k)$, so that for Kac labels of this form we have $a \in \mathcal{I}_{\mathrm{R}}$ and fields with conformal weights $\left(h_{a}, h_{a}\right)$ occur with multiplicity two, once as $\phi_{a}^{e} \in \mathcal{H}_{F}^{\mathrm{R}, \mathrm{ev}}$ and once as $\phi_{a}^{o} \in \mathcal{H}_{F}^{\mathrm{R}, \text { odd }}$.

For the structure constants we use the notation

$$
\phi_{a}^{\alpha}(x) \phi_{b}^{\beta}(y) \sim \sum_{c \in \mathcal{I}} \hat{C}_{a b}^{(\alpha \beta) c}(x-y)^{\Delta_{c}-\Delta_{a}-\Delta_{b}} \phi_{c}^{\alpha+\beta},
$$

where $\alpha, \beta \in\{e, o\}$ and $\alpha+\beta$ stands for the parity of the product. One solution to the bulk crossing relation (2.12) is given by

$$
\begin{array}{ll}
\hat{C}_{a b}^{(e e) c}=\frac{\lambda_{a}^{e} \lambda_{b}^{e}}{\lambda_{c}^{e}} F_{0 c}\left[\begin{array}{ll}
a & b \\
a & b
\end{array}\right], & \hat{C}_{a b}^{(o o) c}=\frac{\lambda_{a}^{o} \lambda_{b}^{o}}{\lambda_{c}^{e}} e^{\pi i\left(h_{G a}-h_{a}-h_{G}\right)} F_{G c}\left[\begin{array}{cc}
a & b \\
G a & G b
\end{array}\right], \\
\hat{C}_{a b}^{(o e) c}=\frac{\lambda_{a}^{o} \lambda_{b}^{e}}{\lambda_{c}^{o}} F_{0 c}\left[\begin{array}{ll}
a & b \\
a & b
\end{array}\right] F_{a G c}\left[\begin{array}{cc}
G a & b \\
G & c
\end{array}\right], & \hat{C}_{a b}^{(e o) c}=e^{\pi i\left(\left(h_{c}-h_{G c}\right)-\left(h_{b}-h_{G b}\right)\right)} \hat{C}_{b a}^{(o e) c} .
\end{array}
$$

The $\lambda_{i}^{e / o} \in \mathbb{C}^{\times}$are normalisation constants which can be chosen at will. The proof that these constants indeed solve the crossing constraint will be given in [2]. There it will also be shown that one can find a topological defect $F$ with the required properties.

One standard normalisation is to make a choice of square roots

$$
\lambda_{a}^{e}=\left(\left.\hat{C}_{a a}^{(e e) 1}\right|_{\lambda=1}\right)^{-\frac{1}{2}}, \quad \lambda_{a}^{o}=\left(\left.\hat{C}_{a a}^{(o o) 1}\right|_{\lambda=1}\right)^{-\frac{1}{2}} .
$$

With this choice one has $\hat{C}_{a a}^{(e e) 1}=\hat{C}_{a a}^{(o o) 1}=1$. 
This concludes the definition of the fermionic minimal model $F M\left(A_{p-1}, A_{q-1}\right)=$ $F M(p, q)$, where $p, q$ are as in (4.1).

Bosonic subtheory. The bosonic subtheory of $F M(p, q)$ is the restriction to the parity even subspace of $\mathcal{H}_{F}$. This can alternatively be understood as the result of summing over spin structures. Explicitly we have

$$
\mathcal{H}_{F}^{\mathrm{ev}}=\bigoplus_{a \in \mathcal{I}} M_{a} \otimes \bar{M}_{a}
$$

which agrees with the state space of the A-type (bosonic) minimal model $M\left(A_{p-1}, A_{q-1}\right)$. Restricting the structure constants to the even subsector and choosing the normalisation $\lambda_{a}^{e}=S_{0 a} / S_{00}$, the solution (4.5) precisely recovers the bulk structure constants for the A-type models in terms of $F$-matrices as given in [10],

$$
C_{a b}{ }^{c}=\frac{S_{0 a} S_{0 b}}{S_{00} S_{0 c}} F_{0 c}\left[\begin{array}{ll}
a & b \\
a & b
\end{array}\right] .
$$

Note that with these conventions, the coupling to the identity field is not normalised to 1 .

\subsection{D-type fermionic models}

We now consider the theory $\widetilde{F M}(p, q)$ obtained from $F M(p, q)$ by shifting parity in the Ramond sector as in section 2.5. We will assume that $p$ is odd and $q$ is even, which according to (4.1) implies that $q \in 4 \mathbb{Z}$. We will also denote $\widetilde{F M}(p, q)$ as $F M\left(A_{p-1}, D_{q / 2+1}\right)$ which will be justified later by the restriction to the bosonic subtheory. The state space $\widetilde{\mathcal{H}}_{F}$ of $\widetilde{F M}(p, q)$ splits as

$$
\begin{aligned}
\widetilde{\mathcal{H}}_{F}^{\mathrm{NS}, \mathrm{ev}}:=\bigoplus_{a \in \mathcal{I}_{\mathrm{NS}}} M_{a} \otimes \bar{M}_{a}, & \widetilde{\mathcal{H}}_{F}^{\mathrm{R}, \mathrm{ev}}:=\bigoplus_{a \in \mathcal{I}_{\mathrm{R}}} M_{a} \otimes \bar{M}_{G a}, \\
\widetilde{\mathcal{H}}_{F}^{\mathrm{NS}, \mathrm{odd}}:=\bigoplus_{a \in \mathcal{I}_{\mathrm{NS}}} M_{a} \otimes \bar{M}_{G a}, & \widetilde{\mathcal{H}}_{F}^{\mathrm{R}, \mathrm{odd}}:=\bigoplus_{a \in \mathcal{I}_{\mathrm{R}}} M_{a} \otimes \bar{M}_{a} .
\end{aligned}
$$

As opposed to $F M(p, q)$, shifting the antiholomorphic label by $G$ or not no longer corresponds to the field being odd. We therefore label the fields as, for $a \in \mathcal{I}$,

$$
\tilde{\phi}_{a}^{u} \quad: \text { weight }\left(h_{a}, h_{a}\right) \quad \tilde{\phi}_{a}^{s}: \text { weight }\left(h_{a}, h_{G a}\right)
$$

where $u$ stands for "unshifted" and $s$ for "shifted" (referring to the shift by $G$ ). The spin grading of $\tilde{\phi}_{a}^{u / s}$ is $\nu_{a}$ as before, but the parity has changed,

$$
\left|\tilde{\phi}_{a}^{u}\right|=\nu_{a}, \quad\left|\tilde{\phi}_{a}^{s}\right|=\nu_{a}+1
$$

As in the A-type case, for $a=\left(r, \frac{q}{2}\right)$ we have $a \in \mathcal{I}_{\mathrm{R}}, G a=a$, and both $\tilde{\phi}_{a}^{u} \in \widetilde{\mathcal{H}}_{F}^{\mathrm{R}, \text { odd }}$ and $\tilde{\phi}_{a}^{s} \in \widetilde{\mathcal{H}}_{F}^{\mathrm{R}, \mathrm{ev}}$ have conformal weights $\left(h_{a}, h_{a}\right)$, so that these weights occur with multiplicity two. 
The structure constants of $\widetilde{F M}(p, q)$ are obtained from (4.5) by the transformation $(2.20)$ :

$$
\begin{aligned}
\widetilde{C}_{a b}^{(u u) c} & =\frac{\lambda_{a}^{u} \lambda_{b}^{u}}{\lambda_{c}^{u}} F_{0 c}\left[\begin{array}{ll}
a & b \\
a & b
\end{array}\right], & \widetilde{C}_{a b}^{(s s) c} & =\frac{\lambda_{a}^{s} \lambda_{b}^{s}}{\lambda_{c}^{u}}(-1)^{\nu_{a}} e^{\pi i\left(h_{G a}-h_{a}-h_{G}\right)} F_{G c}\left[\begin{array}{cc}
a & b \\
G a & G b
\end{array}\right], \\
\widetilde{C}_{a b}^{(s u) c} & =\frac{\lambda_{a}^{s} \lambda_{b}^{u}}{\lambda_{c}^{s}} F_{0 c}\left[\begin{array}{ll}
a & b \\
a & b
\end{array}\right] F_{a G c}\left[\begin{array}{cc}
G a & b \\
G & c
\end{array}\right], & \widetilde{C}_{a b}^{(u s) c} & =(-1)^{\nu_{a}} e^{\pi i\left(\left(h_{c}-h_{G c}\right)-\left(h_{b}-h_{G b}\right)\right)} \widetilde{C}_{b a}^{(s u) c} .
\end{aligned}
$$

Bosonic subtheory. The even subspace of $\widetilde{\mathcal{H}}_{F}$ is

$$
\widetilde{\mathcal{H}}_{F}^{\mathrm{ev}}=\bigoplus_{a \in \mathcal{I}_{\mathrm{NS}}} M_{a} \otimes \bar{M}_{a} \oplus \bigoplus_{a \in \mathcal{I}_{\mathrm{R}}} M_{a} \otimes \bar{M}_{G a}
$$

which is the state space of the D-type minimal model $M\left(A_{p-1}, D_{q / 2+1}\right)$. Note that since $q \in 4 \mathbb{Z}$, this model is always of $D_{\text {odd }}$-type (a permutation modular invariant). When restricting the structure constants to the even subsector, i.e. to $\phi_{a}^{u}, a \in \mathcal{I}_{\mathrm{NS}}$ and $\phi_{a}^{s}$, $a \in \mathcal{I}_{\mathrm{R}}$, the expression (4.12) reproduces the structure constants of the D-series bosonic Virasoro minimal model found in [11].

Since the comparison requires a bit of calculation, we give some details. The expression in $[11]$ is

$$
\begin{aligned}
C_{i_{\alpha} j_{\beta}}{ }^{m_{\gamma}}= & \exp \left(i \frac{\pi}{2}\left(h_{\omega_{\gamma}}+h_{\omega_{\alpha}}-h_{\omega_{\beta}}+2\left(h_{j}-h_{r}\right)+h_{m}-\bar{h}_{m}-h_{i}+\bar{h}_{i}-h_{j}+\bar{h}_{j}\right)\right) \\
& \times \frac{{ }^{(\omega)} B_{i_{\alpha}}^{\omega_{\alpha}{ }^{(\omega)} B_{j_{\beta}}^{\omega_{\beta}}}}{(\omega) B_{m_{\gamma}}^{\omega_{\gamma}}} F_{\mu_{u} \omega_{\gamma}}\left[\begin{array}{ll}
\mu_{u} & \mu_{u} \\
\omega_{\alpha} & \omega_{\beta}
\end{array}\right] F_{\omega_{\beta} r}\left[\begin{array}{cc}
\omega_{\gamma} & \bar{j} \\
\omega_{\alpha} & j
\end{array}\right] F_{\omega_{\alpha} m}\left[\begin{array}{cc}
\bar{i} & r \\
i & j
\end{array}\right] F_{r \bar{m}}\left[\begin{array}{cc}
\bar{i} & \bar{j} \\
m & \omega_{\gamma}
\end{array}\right] .
\end{aligned}
$$

The notation is as follows. The indices $\alpha, \beta, \gamma$ take values in $\{e, o\}$. The index $i_{e}$ corresponds to the field $\phi_{i}^{u}$ and $i_{o}$ to $\phi_{i}^{s}$. Furthermore, $\omega_{e}=(1,1)$ and $\omega_{o}=G$. For $i_{e}$ we have $\bar{h}_{i}=h_{i}$ and for $i_{o}$ we have $\bar{h}_{i}=h_{G i}$. The label $r$ is given by $r=j$ if $\alpha=e$ and $r=G j$ if $\alpha=o$. The constants ${ }^{(\omega)} B_{i_{\alpha}}{ }^{\omega_{\alpha}}$, etc., are certain bulk-boundary structure constants which will be absorbed into the normalisation coefficients (together with a phase for the $G$-shifted fields),

$$
\lambda_{a}^{u}={ }^{(\omega)} B_{a_{e}}^{\omega_{e}}, \quad \lambda_{a}^{s}={ }^{(\omega)} B_{a_{o}}^{\omega_{o}} e^{i \frac{\pi}{2}\left(h_{a}-h_{G a}-h_{G}\right)} .
$$

The label $\mu_{u}$ is the Kac-label $\left(1, \frac{q}{2}\right)$, which is a fixed point for $G$. The $F$-matrix entry $F_{\mu_{u}} \omega_{\gamma}\left[\begin{array}{ll}\mu_{u} & \mu_{u} \\ \omega_{\alpha} & \omega_{\beta}\end{array}\right]$ is either 0 or 1 , depending on whether the $\mathbb{Z}_{2}$ fusion rules in the OPE are obeyed. This grading rule holds by construction for (4.12), so that this $F$-matrix coefficient can be dropped. One can now check sector by sector that the restriction of (4.12) to the even subsector agrees with (4.14). In sector "su", i.e. for $\alpha=o, \beta=e$, this requires an $F$-matrix identity:

$$
\begin{aligned}
& F_{0 G b}\left[\begin{array}{ll}
G & b \\
G & b
\end{array}\right] F_{G c}\left[\begin{array}{cc}
G a & G b \\
a & b
\end{array}\right] F_{G b G c}\left[\begin{array}{cc}
G a & b \\
c & G
\end{array}\right] e^{\pi i\left(h_{G}+h_{b}-h_{G b}\right)} \\
& =F_{0 c}\left[\begin{array}{ll}
a & b \\
a & b
\end{array}\right] F_{a G c}\left[\begin{array}{cc}
G a & b \\
G & c
\end{array}\right] e^{\pi i\left(\left(h_{G}+h_{a}-h_{G a}\right)-\left(h_{G}+h_{c}-h_{G c}\right)\right)}
\end{aligned}
$$




\begin{tabular}{|c|cc|c|c|}
\hline spinless & \multicolumn{2}{|c|}{ even } & odd \\
fields & \multicolumn{2}{|c|}{ NS } & $\mathrm{R}$ & $\mathrm{R}$ \\
& 1 & $\epsilon$ & $\sigma$ & $\mu$ \\
\hline & & & \\
$h=\bar{h}$ & 0 & $\frac{1}{2}$ & $\frac{1}{16}$ & $\frac{1}{16}$ \\
\hline
\end{tabular}

\begin{tabular}{|c|cc|}
\hline fields & \multicolumn{2}{|c|}{ odd \& NS } \\
w. spin & $\psi$ & $\bar{\psi}$ \\
\hline & & \\
$(h, \bar{h})$ & $\left(\frac{1}{2}, 0\right)$ & $\left(0, \frac{1}{2}\right)$ \\
\hline
\end{tabular}

Table 2. Virasoro primary fields in the fermionic Ising model.

Finally, note that since we restrict to the even subsector, for $\phi_{a}^{u}$ we have $\nu_{a}=0$ while for $\phi_{a}^{s}$ we have $\nu_{a}=1$. Accordingly, the factor $(-1)^{\nu_{a}}$ is equal to 1 in $\widetilde{C}_{a b}^{(s u) c}$ and equal to -1 in $\widetilde{C}_{a b}^{(s s) c}$. In the latter case, the minus sign cancels against $e^{-2 \pi i h_{G}}$.

Finally, let us note that fermionic minimal model $F M(p, q)$ and its parity shifted cousin $\widetilde{F M}(p, q)$ are non-isomorphic (as graded theories) whenever the corresponding $D_{\text {odd }}{ }^{-}$ diagram differs from an $A$-diagram. Since $q \in 4 \mathbb{Z}$, this happens for $q \geq 8$. On the other hand, the fermionic Ising model $F M(3,4)$, which we will treat in detail in the next section, is isomorphic to $\widetilde{F M}(3,4)$.

\section{The Ising model and the free fermion}

The Ising model is the Virasoro minimal model $M(3,4)$ and the relevant data is given in appendix A.1. The fermionic Ising model $F M(3,4)$ is really the theory of the free fermion - the field $\phi_{\epsilon}^{o}=\psi$ is a free holomorphic fermion and $\phi_{1}^{o}=\bar{\psi}$ is a free anti-holomorphic fermion. Altogether, the Virasoro primary fields in the fermionic model are given in table 2.

\subsection{Bulk structure constants}

The structure constants of the free fermion can be read off from (4.5), but in this simple model it is easy to compute them directly from the crossing constraint (2.12), and this is what we will do.

For all fields we set $C_{\phi \phi}^{1}=1$, which fixes all normalisations up to signs. The structure constants of the even fields are those of the Ising model and are of course well-known: up to the symmetry properties (2.14) the only remaining structure constant is

$$
C_{\sigma \sigma}^{\epsilon}=\frac{1}{2}
$$

which also fixes the sign-freedom in the normalisation of $\epsilon$. The structure constants involving even and odd fields in the fermionic model have been considered before and some are given in [6] and [21, section 12.3.3], but these are only partial results and it is not clear how the various signs were chosen nor how the full consistency could be checked. Using our formalism, we state and solve the sewing constraints, and taking the same normalisation of the primaries as [21] we agree with the partial results stated there.

The odd fields in the fermionic model are $\psi, \bar{\psi}$ and $\mu$. Setting $i=j=\psi, k=l=\bar{\psi}$ in bulk crossing relation (2.12) results in $\left(\hat{C}_{\psi \bar{\psi}}{ }^{\epsilon}\right)^{2}=-\hat{C}_{\psi \psi}{ }^{1} \hat{C}_{\bar{\psi}} \frac{1}{\bar{\psi}}=-1$. We link the remaining 
free signs in the normalisation of $\psi$ and $\bar{\psi}$ by setting $\epsilon=i \psi \bar{\psi}$, or, in other words,

$$
\hat{C}_{\psi \bar{\psi}}^{\epsilon}=-i \text {. }
$$

The symmetry properties (2.14) relate any two permutations of the three primaries in a structure constants. Up to such permutations, the remaining structure constants involving two odd fields (and no identity field) are

$$
\hat{C}_{\mu \mu \epsilon}, \quad \hat{C}_{\mu \psi \sigma}, \quad \hat{C}_{\mu \bar{\psi} \sigma} .
$$

To fix their value, we consider the crossing constraint (2.12) for the following four choices of parameters: a) $i=\psi, k=\bar{\psi}, j=\ell=\sigma$ and $q=\epsilon$; b) the same with $j=\ell=\mu$; c) $i=j=\sigma, k=l=\mu, q=\psi ;$ and finally d) the same with $q=\bar{\psi}$,

$$
\begin{aligned}
& \hat{C}_{\psi \bar{\psi}}{ }_{\bar{\psi}} \hat{C}_{\sigma \sigma}^{\epsilon}=-e^{\pi i / 2} \hat{C}_{\psi \sigma}^{\mu} \hat{C}_{\bar{\psi} \sigma}^{\mu}, \\
& \hat{C}_{\psi \bar{\psi}}{ }_{\bar{\psi}}^{\epsilon} \hat{C}_{\mu \mu}^{\epsilon}=e^{\pi i / 2} \hat{C}_{\psi \mu}^{\sigma} \hat{C}_{\bar{\psi} \mu}^{\sigma} \\
& \left(\hat{C}_{\sigma \mu}^{\psi}\right)^{2}=-e^{-\pi i / 2}\left(\hat{C}_{\sigma \sigma}^{1} \hat{C}_{\mu \mu}^{1} F_{0 \epsilon}\left[\begin{array}{ll}
\sigma & \sigma \\
\sigma & \sigma
\end{array}\right] F_{00}\left[\begin{array}{cc}
\sigma & \sigma \\
\sigma & \sigma
\end{array}\right]+\hat{C}_{\sigma \sigma}^{\epsilon} \hat{C}_{\mu \mu}^{\epsilon} F_{\epsilon \epsilon}\left[\begin{array}{ll}
\sigma & \sigma \\
\sigma & \sigma
\end{array}\right] F_{\epsilon 0}\left[\begin{array}{cc}
\sigma & \sigma \\
\sigma & \sigma
\end{array}\right]\right), \\
& \left(\hat{C}_{\sigma \mu}^{\bar{\psi}}\right)^{2}=-e^{\pi i / 2}\left(\hat{C}_{\sigma \sigma}^{1} \hat{C}_{\mu \mu}^{1} F_{0 \epsilon}\left[\begin{array}{cc}
\sigma & \sigma \\
\sigma & \sigma
\end{array}\right] F_{00}\left[\begin{array}{cc}
\sigma & \sigma \\
\sigma & \sigma
\end{array}\right]+\hat{C}_{\sigma \sigma}^{\epsilon} \hat{C}_{\mu \mu}^{\epsilon} F_{\epsilon \epsilon}\left[\begin{array}{ll}
\sigma & \sigma \\
\sigma & \sigma
\end{array}\right] F_{\epsilon 0}\left[\begin{array}{ll}
\sigma & \sigma \\
\sigma & \sigma
\end{array}\right]\right) .
\end{aligned}
$$

Using (2.14) we get the following relations between the structure constants appearing in the above constraints and those listed in (5.3),

$$
\begin{array}{lll}
\hat{C}_{\mu \mu}^{\epsilon}=\hat{C}_{\mu \mu \epsilon}, & \hat{C}_{\psi \sigma}^{\mu}=\hat{C}_{\sigma \mu}^{\psi}=\hat{C}_{\mu \psi \sigma}, & \hat{C}_{\psi \mu}^{\sigma}=e^{-\pi i / 2} \hat{C}_{\mu \psi \sigma}, \\
\hat{C}_{\bar{\psi} \sigma}^{\mu}=\hat{C}_{\sigma \mu} \bar{\psi}=\hat{C}_{\mu \bar{\psi} \sigma}, & \hat{C}_{\bar{\psi} \mu}^{\sigma}=e^{\pi i / 2} \hat{C}_{\mu \bar{\psi} \sigma} .
\end{array}
$$

Then, combining this with (5.4a), (5.4b) and (5.2) shows (in agreement with (5.1))

$$
\hat{C}_{\mu \mu}^{\epsilon}=-\hat{C}_{\sigma \sigma}^{\epsilon}=-\frac{1}{2} .
$$

Substituting this into (5.4c) and (5.4d) gives $\left(\hat{C}_{\sigma \mu}^{\psi}\right)^{2}=\frac{i}{2}$ and $\left(\hat{C}_{\sigma \mu}^{\bar{\psi}}\right)^{2}=-\frac{i}{2}$. Conditions (5.4a)-(5.4d) leave an overall sign in the normalisation of $\psi$ and $\bar{\psi}$ undetermined, and we choose

$$
\hat{C}_{\mu \psi \sigma}=\frac{e^{\pi i / 4}}{\sqrt{2}}, \quad \hat{C}_{\mu \bar{\psi} \sigma}=\frac{e^{-\pi i / 4}}{\sqrt{2}} .
$$

This agrees with the constants $\hat{C}_{\psi \sigma}^{\mu}, \hat{C}_{\bar{\psi} \sigma}^{\mu}, \hat{C}_{\psi \mu}^{\sigma}$ and $\hat{C}_{\bar{\psi} \mu}^{\sigma}$ given in [6, eq. (4)] and [21, eq. (12.68)], as well as with (4.5) (the normalisation constants $\lambda_{a}^{e / o}$ are given in appendix A.1).

Consider for a moment the parity shifted theory $\widetilde{F M}(3,4)$. Its structure constants are related to the ones given above as in (2.20). This turns out to be an equivalent theory, and a choice of parity-grading preserving isomorphism from $\widetilde{F M}(3,4)$ (whose fields we denote by $\widetilde{\sigma}, \widetilde{\psi}$, etc.) to $F M(3,4)$ is

$$
\begin{array}{lll}
\text { NS-sector: } & \text { R-sector: } \\
\widetilde{\epsilon} \mapsto-\epsilon, \quad \widetilde{\psi} \mapsto \psi, \quad \widetilde{\bar{\psi}} \mapsto-\bar{\psi} & \tilde{\sigma} \mapsto \mu, \quad \widetilde{\mu} \mapsto-i \sigma .
\end{array}
$$

This can be interpreted as the action of an invertible interface and agrees with the solution to the (ungraded) fermionic defect classifying algebra called "duality ${ }_{2}$ " in table 8 below. 


\begin{tabular}{|l|r|r|r|}
\hline & $B_{1}^{\epsilon}$ & $B_{1}^{\sigma}$ & $B_{a}^{\mu}$ \\
\hline fixed up & 1 & $2^{1 / 4}$ & 0 \\
\hline fixed down & 1 & $-2^{1 / 4}$ & 0 \\
\hline free & -1 & 0 & $2^{1 / 4}$ \\
\hline free & -1 & 0 & $-2^{1 / 4}$ \\
\hline
\end{tabular}

Table 3. Solutions to the bulk-boundary sewing constraints for the fermionic Ising model.

\subsection{Boundary classifying algebra and boundary conditions}

In the fermionic Ising model case, only the three spinless fields $(\epsilon, \sigma, \mu)$ can couple to a weight zero field on the boundary and of these, the two even fields $(\epsilon, \sigma)$ can only couple to a bosonic field of weight zero while the odd field $(\mu)$ can only couple to a fermionic field of weight zero.

We shall denote a possible fermionic weight zero field by $a$ and normalise its two point function to $\langle a a\rangle=1$, so that $c_{a a}^{1}=1$. As a consequence, we would like to find the sewing constraints on the following set of bulk-boundary structure constants:

$$
B_{1}^{\epsilon}, B_{1}^{\sigma}, B_{a}^{\mu},
$$

and we have six sewing constraints of the form (3.9), namely

$$
\begin{aligned}
B_{1}^{\epsilon} B_{1}^{\epsilon} & =\hat{C}_{\epsilon \epsilon}^{1} F_{00}\left[\begin{array}{ll}
\epsilon & \epsilon \\
\epsilon
\end{array}\right]=1, \\
B_{1}^{\epsilon} B_{1}^{\sigma} & =\hat{C}_{\epsilon \sigma}^{\sigma} F_{\sigma 0}\left[\begin{array}{cc}
\sigma & \sigma \\
\epsilon & \epsilon
\end{array}\right] B_{1}^{\sigma}=B_{1}^{\sigma}, \\
B_{1}^{\epsilon} B_{a}^{\mu} & =\hat{C}_{\epsilon \mu}^{\mu} F_{\sigma 0}\left[\begin{array}{cc}
\sigma & \sigma \\
\epsilon & \epsilon
\end{array}\right] B_{1}^{\mu}=-B_{a}^{\mu}, \\
B_{1}^{\sigma} B_{1}^{\sigma} & =\hat{C}_{\sigma \sigma}^{1} F_{00}\left[\begin{array}{cc}
\sigma & \sigma \\
\sigma & \sigma
\end{array}\right]+\hat{C}_{\sigma \sigma}^{\epsilon} F_{\epsilon 0}\left[\begin{array}{ll}
\sigma & \sigma \\
\sigma & \sigma
\end{array}\right] B_{1}^{\epsilon}=\frac{1}{\sqrt{2}}\left(1+B_{1}^{\epsilon}\right), \\
B_{1}^{\sigma} B_{a}^{\mu} & =0, \\
B_{a}^{\mu} B_{a}^{\mu} & =\hat{C}_{\mu \mu}^{1} F_{00}\left[\begin{array}{cc}
\sigma & \sigma \\
\sigma & \sigma
\end{array}\right]+\hat{C}_{\mu \mu}^{\epsilon} F_{\epsilon 0}\left[\begin{array}{ll}
\sigma & \sigma \\
\sigma & \sigma
\end{array}\right] B_{1}^{\epsilon}=\frac{1}{\sqrt{2}}\left(1-B_{1}^{\epsilon}\right) .
\end{aligned}
$$

These equations have four solutions, given in table 3, but the last two can be identified by the change of normalisation $a \rightarrow-a$, and so there are three inequivalent solutions to the boundary sewing constraints, two fixed boundary conditions which do not require a fermionic weight zero field, and one free boundary condition which does require a fermionic weight zero field.

From (5.10) we can also read off the fermionic boundary classifying algebra $\hat{\mathcal{B}}$ in $(3.11)$ for the free fermion:

$$
\begin{aligned}
& B^{\epsilon} B^{\epsilon}=1, \quad B^{\sigma} B^{\sigma}=\frac{1}{\sqrt{2}}\left(1+B^{\epsilon}\right), \quad B^{\mu} B^{\mu}=\frac{1}{\sqrt{2}}\left(1-B^{\epsilon}\right), \\
& B^{\epsilon} B^{\sigma}=B^{\sigma}, \quad B^{\epsilon} B^{\mu}=-B^{\mu}, \quad B^{\sigma} B^{\mu}=0 \text {. }
\end{aligned}
$$


This is a super-algebra and the parity of each generator agrees with that of the corresponding bulk field: $B^{\epsilon}, B^{\sigma}$ are even and $B^{\mu}$ is odd. Now we can more easily identify the elementary boundary conditions by expressing $\hat{\mathcal{B}}$ as a direct sum of copies of $\mathbb{C}$ and the Clifford algebra $C \ell_{1}$ which correspond directly to the elementary boundary conditions without overcounting the free boundary condition (cf. the discussion in section 3.1.4),

$$
\hat{\mathcal{B}}=\mathbb{C} e_{+} \oplus \mathbb{C} e_{-} \oplus\left(\mathbb{C} f_{f} \oplus \mathbb{C} a_{f}\right),
$$

where the indices,,$+- f$ correspond to fixed up, fixed down and free. Explicitly,

$$
\begin{aligned}
& e_{+}=\frac{1}{4}\left(1+B^{\epsilon}\right)+2^{-3 / 4} B^{\sigma}, \quad e_{-}=\frac{1}{4}\left(1+B^{\epsilon}\right)-2^{-3 / 4} B^{\sigma}, \\
& f_{f}=\frac{1}{2}\left(1-B^{\epsilon}\right), \quad a_{f}=2^{-1 / 4} B^{\mu} .
\end{aligned}
$$

One can check that these satisfy the algebra (3.13) and from the action of the generators $B^{\epsilon}, B^{\sigma}$ and $B^{\mu}$, we can read off the representations:

\begin{tabular}{|l|c|c|c|}
\hline & $B^{\epsilon}$ & $B^{\sigma}$ & $B^{\mu}$ \\
\hline fixed up & 1 & $2^{1 / 4}$ & 0 \\
\hline fixed down & 1 & $-2^{1 / 4}$ & 0 \\
\hline free & $-\mathbb{1}$ & 0 & $2^{1 / 4} \cdot \mathbb{A}$ \\
\hline
\end{tabular}

where the $2 \times 2$ matrices $\mathbb{1}$ and $\mathbb{A}$ are

$$
\mathbb{1}=\left(\begin{array}{ll}
1 & 0 \\
0 & 1
\end{array}\right), \quad \mathbb{A}=\left(\begin{array}{ll}
0 & 1 \\
1 & 0
\end{array}\right) .
$$

It is easy to see that the matrix representation of the free boundary condition is reducible and (by diagonalising $\mathbb{A}$ ) splits into exactly the two solution in table 3 but this does not respect the even-odd nature of the generators and leads to the over-counting of the free boundary condition in table 3 ; only when ensuring that the representation of $\hat{\mathcal{B}}$ respects the even-odd grading do we get the correct counting (cf. (3.15) for the precise statement).

\subsection{Boundary field content and boundary states}

It is also instructive to consider the contribution of non-zero weight boundary fields as this will determine the full boundary field content of each boundary condition as well as give the gluing conditions for the fermion fields on the boundaries.

\subsubsection{Boundary field content}

Given the bulk field content in the fermionic Ising model, one only has to consider $h=\frac{1}{2}$ boundary fields as no other couplings are possible.

The first result is that there must always exist a fermionic weight $\frac{1}{2}$ field, which we denote $\mu_{B}$ and which could be identified with the boundary disorder field. The reason is that the bulk fermionic fields can only couple to a weight $\frac{1}{2}$ field on the boundary. If we 


\begin{tabular}{|c|c|c|c|c|c|c|c|}
\hline & $B_{1}^{\epsilon}$ & $B_{1}^{\sigma}$ & $B_{\sigma_{B}}^{\sigma}$ & $B_{a}^{\mu}$ & $B_{\mu_{B}}^{\mu}$ & $B_{\mu_{B}}^{\psi}$ & $B_{\mu_{B}}^{\bar{\psi}}$ \\
\hline$B_{1}^{\epsilon}$ & 1 & & & & & & \\
\hline$B_{1}^{\sigma}$ & $B_{1}^{\sigma}$ & $\frac{1}{\sqrt{2}}\left(1+B_{1}^{\epsilon}\right)$ & & & & & \\
\hline$B_{\sigma_{B}}^{\sigma}$ & $-B_{\sigma_{B}}^{\sigma}$ & 0 & $\frac{1}{2 \sqrt{2}}\left(1-B_{1}^{\epsilon}\right)$ & & & & \\
\hline$B_{a}^{\mu}$ & $-B_{a}^{\mu}$ & 0 & $\frac{1}{2}\left(B_{\mu_{B}}^{\bar{\psi}}-B_{\mu_{B}}^{\psi}\right)$ & $\frac{1}{\sqrt{2}}\left(1-B_{1}^{\epsilon}\right)$ & & & \\
\hline$B_{\mu_{B}}^{\mu}$ & $B_{\mu_{B}}^{\mu}$ & $\frac{1}{2}\left(B_{\mu_{B}}^{\psi}+B_{\mu_{B}}^{\bar{\psi}}\right)$ & 0 & 0 & $\frac{1}{2 \sqrt{2}}\left(1+B_{1}^{\epsilon}\right)$ & & \\
\hline$B_{\mu_{B}}^{\psi}$ & $B_{\mu_{B}}^{\bar{\psi}}$ & $\sqrt{2} B_{\mu_{B}}^{\mu}$ & $-\frac{1}{\sqrt{2}} B_{a}^{\mu}$ & $-\sqrt{2} B_{\sigma_{B}}^{\sigma}$ & $\frac{1}{\sqrt{2}} B_{1}^{\sigma}$ & 1 & \\
\hline$B_{\mu_{B}}^{\bar{\psi}}$ & $B_{\mu_{B}}^{\psi}$ & $\sqrt{2} B_{\mu_{B}}^{\mu}$ & $\frac{1}{\sqrt{2}} B_{a}^{\mu}$ & $\sqrt{2} B_{\sigma_{B}}^{\sigma}$ & $\frac{1}{\sqrt{2}} B_{1}^{\sigma}$ & $B_{1}^{\epsilon}$ & 1 \\
\hline
\end{tabular}

Table 4. The sewing constraints from (3.7), rewritten in the from $B_{a}^{\alpha} B_{b}^{\beta}=\sum_{\gamma, c} C_{\alpha a, \beta b}{ }^{\gamma c} B_{c}^{\gamma}$ and stated explicitly as a multiplication table; the sewing constraints for the standard Ising model are in the top left $3 \times 3$ sub-table.

denote the bulk-boundary coupling of $\psi$ as $B_{\mu_{B}}^{\psi}$ and if the boundary field two point function normalisation is $c_{\mu_{B} \mu_{B}}{ }^{1}$, then the sewing constraint (3.7) requires $\left(B_{\mu_{B}}^{\psi}\right)^{2} c_{\mu_{B} \mu_{B}}{ }^{1}=1$ and hence we see that not only must such a field exist but that the bulk fermions must couple to it. We shall not consider the possibility of two distinct boundary fermions but instead suppose that both bulk fields $\psi$ and $\bar{\psi}$ couple to the same boundary field.

The next question is whether there is also an even (bosonic) weight $\frac{1}{2}$ boundary field $\sigma_{B}$ (which could be identified with the boundary spin). If there is such a field then its OPE with $\mu_{B}$ must necessarily be to an odd weight 0 field and conversely the OPE of an odd weight zero field with $\mu_{B}$ must be an even weight $\frac{1}{2}$ field $\sigma_{B}$. If we normalise $a^{2}=c_{\sigma_{B} \sigma_{B}}{ }^{1}=$ $c_{\mu_{B} \mu_{B}}{ }^{1}=1$ then the OPE algebra is forced by the boundary sewing constraints to have the form

$$
a \sigma_{B}=\lambda \mu_{B}, \quad a \mu_{B}=(1 / \lambda) \sigma_{B}, \quad \sigma_{B} a=(1 / \lambda) \mu_{B}, \quad \mu_{B} a=\lambda \sigma_{B},
$$

where $\lambda^{4}=1$. The values $\lambda= \pm 1$ turn out to be inconsistent with the bulk-boundary sewing constraints and $\lambda= \pm i$ are equivalent under a field redefinition, hence we make the choice $\lambda=i$ from here on. With this boundary field algebra and the seven structure constants

$$
B_{1}^{\epsilon}, B_{1}^{\sigma}, B_{a}^{\mu}, B_{\sigma_{B}}^{\sigma}, B_{\mu_{B}}^{\mu}, B_{\mu_{B}}^{\psi}, B_{\mu_{B}}^{\bar{\psi}},
$$

there are 28 sewing constraints coming from (3.7). These amount to 28 multiplication rules for the structure constants, which are given in table 4 .

Viewed as equations, the sewing constraints have 8 solutions, four fixed with $B_{1}^{\epsilon}=1$ and four free with $B_{1}^{\epsilon}=-1$. The eight solutions are given in table 5 . In the four fixed 


\begin{tabular}{|c|c|c|c|c|c|c|c|}
\hline & $B_{1}^{\epsilon}$ & $B_{1}^{\sigma}$ & $B_{\sigma_{B}}^{\sigma}$ & $B_{a}^{\mu}$ & $B_{\mu_{B}}^{\mu}$ & $B_{\mu_{B}}^{\psi}$ & $B_{\mu_{B}}^{\bar{\psi}}$ \\
\hline fixed up & 1 & $2^{1 / 4}$ & 0 & 0 & $2^{-1 / 4}$ & 1 & 1 \\
\hline fixed up & 1 & $2^{1 / 4}$ & 0 & 0 & $-2^{-1 / 4}$ & -1 & -1 \\
\hline fixed down & 1 & $-2^{1 / 4}$ & 0 & 0 & $-2^{-1 / 4}$ & 1 & 1 \\
\hline fixed down & 1 & $-2^{1 / 4}$ & 0 & 0 & $2^{-1 / 4}$ & -1 & -1 \\
\hline free & -1 & 0 & $2^{-1 / 4}$ & $2^{1 / 4}$ & 0 & -1 & 1 \\
\hline free & -1 & 0 & $2^{-1 / 4}$ & $-2^{1 / 4}$ & 0 & 1 & -1 \\
\hline free & -1 & 0 & $-2^{-1 / 4}$ & $2^{1 / 4}$ & 0 & 1 & -1 \\
\hline free & -1 & 0 & $-2^{-1 / 4}$ & $-2^{1 / 4}$ & 0 & -1 & 1 \\
\hline
\end{tabular}

Table 5. The eight solutions to the full sewing constraints with $\lambda=i$; the three solutions to the sewing constraints for the standard Ising model are given by the first three columns of the table.

\begin{tabular}{|c|c|c|c|c|c|c|c|}
\hline & $B_{1}^{\epsilon}$ & $B_{1}^{\sigma}$ & $B_{\sigma_{B}}^{\sigma}$ & $B_{a}^{\mu}$ & $B_{\mu_{B}}^{\mu}$ & $B_{\mu_{B}}^{\psi}$ & $B_{\mu_{B}}^{\bar{\psi}}$ \\
\hline fixed up & $\mathbb{1}$ & $2^{1 / 4} \cdot \mathbb{1}$ & 0 & 0 & $2^{-1 / 4} \cdot \mathbb{A}$ & $\mathbb{A}$ & $\mathbb{A}$ \\
\hline fixed down & $\mathbb{1}$ & $-2^{1 / 4} \cdot \mathbb{1}$ & 0 & 0 & $2^{-1 / 4} \cdot \mathbb{A}$ & $-\mathbb{A}$ & $-\mathbb{A}$ \\
\hline free & $-\mathbb{1}$ & 0 & $2^{-1 / 4} \cdot \mathbb{1}$ & $2^{1 / 4} \cdot \mathbb{A}$ & 0 & $-\mathbb{A}$ & $\mathbb{A}$ \\
\hline free & $-\mathbb{1}$ & 0 & $-2^{-1 / 4} \cdot \mathbb{1}$ & $2^{1 / 4} \cdot \mathbb{A}$ & 0 & $\mathbb{A}$ & $-\mathbb{A}$ \\
\hline
\end{tabular}

Table 6. Representations of the boundary classifying super-algebra of the fermionic Ising model.

solutions, there are no couplings to the fields $a$ and $\sigma_{B}$ and so these fields can be consistently excluded from the set of boundary fields, as expected.

Only three of the eight solutions are physically distinct. Namely, for each of the sign choices $\zeta, \xi \in\{ \pm 1\}$ we can redefine the boundary fields as $a \mapsto \zeta a, \sigma_{B} \rightarrow \xi \sigma_{B}, \mu_{B} \rightarrow \zeta \xi \mu_{B}$. This agrees with the three physically distinct solutions found in table 3 .

What is perhaps surprising is that the full set of sewing constraints in table 4 also defines a commutative algebra [with identity] with generators $\left\{1, B_{1}^{\epsilon}, B_{1}^{\sigma}, B_{a}^{\mu}, B_{\sigma_{B}}^{\sigma}, B_{\mu_{B}}^{\mu}\right.$, $\left.B_{\mu_{B}}^{\psi}, B_{\mu_{B}}^{\bar{\psi}}\right\}$, with the boundary classifying algebra as a sub-algebra, and the solutions in table 5 are the eight one-dimensional representations of this algebra.

As with the classifying algebra, this commutative algebra can also be viewed as a superalgebra with even generators $\left\{1, B_{1}^{\epsilon}, B_{1}^{\sigma}, B_{\sigma_{B}}^{\sigma}\right\}$, and odd generators $\left\{B_{a}^{\mu}, B_{\mu_{B}}^{\mu}, B_{\mu_{B}}^{\psi}, B_{\mu_{B}}^{\bar{\psi}}\right\}$, and the eight one-dimensional representations in table 5 combine into four representations of this super-algebra on $\mathbb{C}^{1 \mid 1}$, of which the final two are again related by $\sigma_{B} \rightarrow-\sigma_{B}, \mu_{B} \rightarrow$ $-\mu_{B}$ and so are equivalent physically; these are given in table 6 .

This table also shows that the fermions have opposite gluing conditions on fixed and free boundary conditions. On the free boundary condition with $B^{\epsilon}=-1$, the bulk fermions obey $\psi=-\bar{\psi}$ on the boundary; on the fixed boundary conditions they obey $\psi=\bar{\psi}$. 


\subsubsection{Boundary states}

Since we are working with theories on a fixed spin structure, each boundary condition $b$ will be associated to two boundary states, one state $|b\rangle_{\text {NS }}$ entirely in the NS sector and one state $|b\rangle_{\mathrm{R}}$ entirely in the $\mathrm{R}$ sector. In our approach, correlators are overall even linear forms, so that a one-point correlator on a disc is an even linear map $\mathcal{H}_{F} \rightarrow \mathbb{C}$. This implies that boundary states are purely even.

The bulk-boundary couplings to the identity field (cf. table 3 ) determine the overlap of the boundary states with each primary bulk field, normalised by the overlap with the vacuum. Namely, for primary bulk fields $\phi_{i}$ in the NS sector and $\phi_{j}$ in the R sector we have

$$
\frac{\mathrm{NS}\left\langle b \mid \phi_{i}\right\rangle}{{ }_{\mathrm{NS}}\langle b \mid 0\rangle}=B_{1}^{i}, \quad \frac{\mathrm{R}\left\langle b \mid \phi_{j}\right\rangle}{\mathrm{NS}_{\mathrm{N}}\langle b \mid 0\rangle}=B_{1}^{j} .
$$

This fixes the expansion of $|b\rangle_{\mathrm{NS}, \mathrm{R}}$ in terms of Virasoro-Ishibashi states $\left.\left.\left.|1\rangle\right\rangle,|\sigma\rangle\right\rangle,|\epsilon\rangle\right\rangle$ up to the overall constant $\langle 0 \mid b\rangle_{N S}$.

To determine $\langle 0 \mid b\rangle_{\mathrm{NS}}$ (up to a sign) we will make use of the fact that we know the boundary field content for each boundary condition:

$$
\text { fixed }: 1, \mu_{B}, \quad \text { free }: 1, a, \mu_{B}, \sigma_{B} .
$$

This means that the NS boundary states have to satisfy

$$
\begin{aligned}
\mathrm{NS}_{\mathrm{N}}\left\langle\text { fixed }\left|q^{L_{0}+\bar{L}_{0}-1 / 24}\right| \text { fixed }\right\rangle_{\mathrm{NS}} & =\chi_{0}(\tilde{q})+\chi_{1 / 2}(\tilde{q}), \\
\mathrm{NS}\left\langle\text { free }\left|q^{L_{0}+\bar{L}_{0}-1 / 24}\right| \text { free }\right\rangle_{\mathrm{NS}} & =2 \chi_{0}(\tilde{q})+2 \chi_{1 / 2}(\tilde{q}),
\end{aligned}
$$

where as usual $q=\exp (2 \pi i \tau), \tilde{q}=\exp (-2 \pi i / \tau)$ and $\chi_{h}$ are the characters of the Virasoro highest weight representations of weight $h$.

Putting all this together, we arrive at (up to an overall undetermined sign for each boundary condition)

$$
\begin{aligned}
\mid \text { fixed }+\rangle_{\mathrm{NS}} & =|0\rangle\rangle+|\epsilon\rangle\rangle, & \mid \text { fixed }+\rangle_{\mathrm{R}} & \left.=2^{1 / 4}|\sigma\rangle\right\rangle, \\
\mid \text { fixed }-\rangle_{\mathrm{NS}} & =|0\rangle\rangle+|\epsilon\rangle\rangle, & \mid \text { fixed }-\rangle_{\mathrm{R}} & \left.=-2^{1 / 4}|\sigma\rangle\right\rangle, \\
\mid \text { free }\rangle_{\mathrm{NS}} & =\sqrt{2}(|0\rangle\rangle-|\epsilon\rangle\rangle), & \mid \text { free }\rangle_{\mathrm{R}} & =0 .
\end{aligned}
$$

The overlaps between the R-sector boundary states must give the supertrace over the field contents, and the above states correctly give these supertraces,

$$
\begin{aligned}
{ }_{R}\left\langle\text { fixed }\left|q^{L_{0}+\bar{L}_{0}-1 / 24}\right| \text { fixed }\right\rangle_{R} & =\chi_{0}(\tilde{q})-\chi_{1 / 2}(\tilde{q}), \\
{ }_{R}\left\langle\text { free }\left|q^{L_{0}+\bar{L}_{0}-1 / 24}\right| \text { free }\right\rangle_{R} & =0 .
\end{aligned}
$$

As briefly mentioned in the end of section 3.1.4, one can think of boundary states as coming in pairs which differ by an overall sign; the three boundary states presented above are then characterised by the condition $\langle 0 \mid b\rangle_{\mathrm{NS}}>0$.

Note that the NS components in (5.21) agree with the conjectures in [12] for the NS-sector of the boundary states, although that paper does not correctly account for the fermionic fields in this model nor the Ramond sectors. 


\begin{tabular}{|c|c|c|c|c|c|c|c|}
\hline & $D_{1}^{\epsilon \epsilon}$ & $D_{1}^{\sigma \sigma}$ & $D_{1}^{\mu \mu}$ & $D_{a}^{\sigma \mu}$ & $D_{a}^{\mu \sigma}$ & $D_{1}^{\psi \psi}$ & $D_{1}^{\bar{\psi} \bar{\psi}}$ \\
\hline$D_{1}^{\epsilon \epsilon}$ & 1 & & & & & & \\
\hline$D_{1}^{\sigma \sigma}$ & $D_{1}^{\sigma \sigma}$ & $\frac{1}{2}\left(1+D_{1}^{\epsilon \epsilon}\right)$ & & & & & \\
\hline$D_{1}^{\mu \mu}$ & $D_{1}^{\mu \mu}$ & $\frac{1}{2}\left(D_{1}^{\psi \psi}+D_{1}^{\bar{\psi} \bar{\psi}}\right)$ & $\frac{1}{2}\left(1+D_{1}^{\epsilon \epsilon}\right)$ & & & & \\
\hline$D_{a}^{\sigma \mu}$ & $-D_{a}^{\sigma \mu}$ & 0 & 0 & $\frac{1}{2}\left(-1+D_{1}^{\epsilon \epsilon}\right)$ & & & \\
\hline$D_{a}^{\mu \sigma}$ & $-D_{a}^{\mu \sigma}$ & 0 & 0 & $-\frac{i}{2}\left(D_{1}^{\psi \psi}-D_{1}^{\bar{\psi} \bar{\psi}}\right)$ & $\frac{1}{2}\left(1-D_{1}^{\epsilon \epsilon}\right)$ & & \\
\hline$D_{1}^{\psi \psi} \psi$ & $D_{1}^{\bar{\psi} \bar{\psi}}$ & $D_{1}^{\mu \mu}$ & $D_{1}^{\sigma \sigma}$ & $-i D_{a}^{\mu \sigma}$ & $i D_{a}^{\sigma \mu}$ & 1 & \\
\hline$D_{1}^{\bar{\psi} \bar{\psi}}$ & $D_{1}^{\psi \psi}$ & $D_{1}^{\mu \mu}$ & $D_{1}^{\sigma \sigma}$ & $i D_{a}^{\mu \sigma}$ & $-i D_{a}^{\sigma \mu}$ & $D_{1}^{\epsilon \epsilon}$ & 1 \\
\hline
\end{tabular}

Table 7. The defect classifying algebra for the fermionic Ising model.

Boundary states in the NS-sector of free fermions are also considered in [22], and the boundary states for a single Majorana fermion given in appendix D there agree with the NS-component in (5.21) up to normalisation.

Finally, we note that our boundary states are related to the boundary states for the purely bosonic Ising model by taking a superposition of the NS- and R-sector boundary states,

$$
|a\rangle_{\text {Ising }}=\frac{1}{\sqrt{2}}\left(|a\rangle_{\mathrm{NS}}+|a\rangle_{\mathrm{R}}\right)
$$

\subsection{Defect classifying algebra and defect operators}

\subsubsection{Classifying algebra and defect conditions}

We consider now the defect classifying algebra of the standard Ising model and the fermionic version, assuming that there is a single bosonic field of weight zero (denoted 1 ) on the defect and at most one fermionic defect field of weight zero denoted $a$ which satisfies $a^{2}=1$. The bulk-defect structure constants for the non-identity bulk fields in the Ising case are $\left\{D_{1}^{\epsilon \epsilon}, D_{1}^{\sigma \sigma}\right\}$, and for the fermionic case they are $\left\{D_{1}^{\epsilon \epsilon}, D_{1}^{\sigma \sigma}, D_{1}^{\mu \mu}, D_{a}^{\sigma \mu}, D_{a}^{\mu \sigma}, D_{1}^{\psi \psi}, D_{1}^{\bar{\psi} \bar{\psi}}\right\}$. The sewing constraints are given by the multiplication table 7 .

Viewed as equations, there are eight solutions to the defect sewing constraints, given in table 8 .

We can view the bulk-defect structure constants as algebra generators of a superalgebra $\hat{\mathcal{D}}$, the fermionic defect classifying algebra, with odd generators $\left\{D_{a}^{\sigma \mu}, D_{a}^{\mu \sigma}\right\}$ and the rest being even. As an ungraded algebra $\hat{\mathcal{D}}$ is commutative, and the solutions in table 8 are its eight one-dimensional representations. When viewed as representations of the superalgebra, the first four are one-dimensional representations on $\mathbb{C}^{1 / 0}$ while the second four combine to form two two-dimensional representations on $\mathbb{C}^{1 \mid 1}$. Accordingly, $\hat{\mathcal{D}}$ decomposes into four copies of $\mathbb{C}$ (with generators $e_{\alpha}$ ) and two copies of $C \ell_{1}$ (with generators $\left\{f_{\alpha}, a_{\alpha}\right\}$ ) 


\begin{tabular}{|c|c|c|c|c|c|c|c|}
\hline & $D_{1}^{\epsilon \epsilon}$ & $D_{1}^{\sigma \sigma}$ & $D_{1}^{\mu \mu}$ & $D_{1}^{\psi \psi}$ & $D_{1}^{\bar{\psi} \bar{\psi}}$ & $D_{a}^{\sigma \mu}$ & $D_{a}^{\mu \sigma}$ \\
\hline identity $^{\prime}$ & 1 & 1 & 1 & 1 & 1 & 0 & 0 \\
\hline identity $^{\prime}$ & 1 & 1 & -1 & -1 & -1 & 0 & 0 \\
\hline spin reversal & 1 & -1 & -1 & 1 & 1 & 0 & 0 \\
\hline spin reversal $^{\prime}$ & 1 & -1 & 1 & -1 & -1 & 0 & 0 \\
\hline duality $^{1}$ & -1 & 0 & 0 & 1 & -1 & $i$ & -1 \\
\hline duality $^{2}$ & -1 & 0 & 0 & 1 & -1 & $-i$ & 1 \\
\hline duality $^{3}$ & -1 & 0 & 0 & -1 & 1 & $-i$ & -1 \\
\hline duality $^{4}$ & -1 & 0 & 0 & -1 & 1 & $i$ & 1 \\
\hline
\end{tabular}

Table 8. Solutions for the bulk-defect structure constants in the fermionic Ising model.

satisfying the same algebra (3.13). The explicit forms are:

$$
\begin{aligned}
e_{\mathrm{id}}= & \frac{1}{8}\left(1+D_{1}^{\epsilon \epsilon}+2 D_{1}^{\mu \mu}+2 D_{1}^{\sigma \sigma}+D_{1}^{\bar{\psi} \bar{\psi}}+D_{1}^{\psi \psi}\right), \\
e_{i d^{\prime}}= & \frac{1}{8}\left(1+D_{1}^{\epsilon \epsilon}-2 D_{1}^{\mu \mu}+2 D_{1}^{\sigma \sigma}-D_{1}^{\bar{\psi} \bar{\psi}}-D_{1}^{\psi \psi}\right), \\
e_{s}= & \frac{1}{8}\left(1+D_{1}^{\epsilon \epsilon}-2 D_{1}^{\mu \mu}-2 D_{1}^{\sigma \sigma}+D_{1}^{\bar{\psi} \bar{\psi}}+D_{1}^{\psi \psi}\right), \\
e_{s^{\prime}}= & \frac{1}{8}\left(1+D_{1}^{\epsilon \epsilon}+2 D_{1}^{\mu \mu}-2 D_{1}^{\sigma \sigma}-D_{1}^{\bar{\psi} \bar{\psi}}-D_{1}^{\psi \psi}\right), \\
f_{d 12}= & \frac{1}{4}\left(1-D_{1}^{\epsilon \epsilon}-D_{1}^{\bar{\psi} \bar{\psi}}+D_{1}^{\psi \psi}\right), \quad a_{d 12}=\frac{1}{2}\left(D_{a}^{\mu \sigma}+i D_{a}^{\sigma \mu}\right), \\
f_{d 34}= & \frac{1}{4}\left(1-D_{1}^{\epsilon \epsilon}+D_{1}^{\bar{\psi} \bar{\psi}}-D_{1}^{\psi \psi}\right), \quad a_{d 34}=\frac{1}{2}\left(D_{a}^{\mu \sigma}-i D_{a}^{\sigma \mu}\right) .
\end{aligned}
$$

\subsubsection{Defect field content and defect operators}

Topological defects can also be described by operators on the Hilbert space of the theory which commute with the Virasoro algebra, and hence are sums of intertwiners between equivalent Virasoro representations. The bulk-defect structure constants are proportional to the coefficients of these intertwiners and hence the defect operator is defined, up to an overall scalar multiple, by the bulk-defect structure constants. This overall multiple can itself be fixed (up to a sign) by the requirement that it correctly determines the field content on the defect, and in particular that it correctly determines the dimension of the space of zero-weight fields on the defect.

We illustrate this in the case of the bosonic and fermionic Ising defects. The bosonic Hilbert space is

$$
\mathcal{H}_{\text {Ising }}=\left(M_{0} \otimes \bar{M}_{0}\right) \oplus\left(M_{\epsilon} \otimes \bar{M}_{\epsilon}\right) \oplus\left(M_{\sigma} \otimes \bar{M}_{\sigma}\right),
$$


where $M_{a}$ denotes the corresponding irreducible Virasoro representation. This means a topological defect operator takes the form

$$
\hat{D}=\alpha P_{00}+\beta P_{\epsilon \epsilon}+\gamma P_{\sigma \sigma}
$$

The constants $D_{1}^{i j}$ are given by

$$
D_{1}^{i j}=\frac{\langle j|\hat{D}| i\rangle}{\langle 0|\hat{D}| 0\rangle}
$$

and hence the defect is fixed up to a scalar,

$$
\hat{D}=\lambda\left(P_{00}+D_{1}^{\epsilon \epsilon} P_{\epsilon \epsilon}+D_{1}^{\sigma \sigma} P_{\sigma \sigma}\right),
$$

The field content on the defect is then given by the modular transform of the trace on the cylinder,

$$
Z(q, \bar{q})=\operatorname{Tr}\left(q^{L_{0}+\bar{L}_{0}-c / 12} D \cdot D^{\dagger}\right)=\lambda^{2}\left(\left|\chi_{0}(q)\right|^{2}+\left(D_{1}^{\epsilon \epsilon}\right)^{2}\left|\chi_{1 / 2}(q)\right|^{2}+\left(D_{1}^{\sigma \sigma}\right)^{2}\left|\chi_{1 / 16}(q)\right|^{2}\right) .
$$

The upshot is that $\lambda= \pm 1$ for the identity and spin defects and $\pm \sqrt{2}$ for the duality defect, cf. [23, 24].

The same ideas can be applied to the defects in the fermionic model, with the observation that the defects come in two versions, depending on the spin structure, so that there are separate defect operators acting on the Neveu-Schwarz and Ramond sectors of the Hilbert space. In the fermionic model the Hilbert space is

$$
\begin{aligned}
\mathcal{H}_{F} & =\mathcal{H}_{F}^{\mathrm{NS}}+\mathcal{H}_{F}^{R}, \\
\mathcal{H}_{F}^{\mathrm{NS}} & =\left(M_{0}+M_{1 / 2}\right) \otimes\left(\bar{M}_{0}+\bar{M}_{1 / 2}\right), \quad \mathcal{H}_{F}^{R}=\left.\left.M_{1 / 16} \otimes \bar{M}_{1 / 16}\right|_{\sigma} \oplus M_{1 / 16} \otimes \bar{M}_{1 / 16}\right|_{\mu} .
\end{aligned}
$$

The defect operators in the two sectors are then given by the bulk-defect structure constants up to an overall constant

$$
\hat{D}^{\mathrm{NS}}=\lambda\left(P_{0}+D_{1}^{\psi \psi} P_{\psi}+D_{1}^{\bar{\psi} \bar{\psi}} P_{\bar{\psi}}+D_{1}^{\epsilon \epsilon} P_{\epsilon}\right), \hat{D}^{R}=\lambda\left(D_{1}^{\sigma \sigma} P_{\sigma}+D_{1}^{\mu \mu} P_{\mu}\right)
$$

The main difference is that the space of weight zero fields on the defects can now be either one or two, depending on whether the defect supports the fermionic weight zero field $a$ or not.

When we perform the calculations we find that $\hat{D}^{\mathrm{NS}}$ are in fact identical with the defect operators proposed in [12]. We give the explicit forms in table 9.

We note that the defects of the bosonic Ising model are given by the combinations

$$
D_{\mathrm{id}}^{\mathrm{Ising}}=\frac{1}{2}\left(D_{\mathrm{id}}+D_{i d^{\prime}}\right), \quad D_{\mathrm{spin}}^{\mathrm{Ising}}=\frac{1}{2}\left(D_{s}+D_{s^{\prime}}\right), \quad D_{\text {duality }}^{\mathrm{Ising}}=\frac{1}{2}\left(D_{d 12}+D_{d 34}\right) .
$$




\begin{tabular}{|c|c|c|}
\hline & $\mathrm{NS}$ & $\mathrm{R}$ \\
\hline $\mathrm{id}$ & $P_{0}+P_{\psi}+P_{\bar{\psi}}+P_{\epsilon}$ & $P_{\sigma}+P_{\mu}$ \\
\hline $\mathrm{id}^{\prime}$ & $P_{0}-P_{\psi}-P_{\bar{\psi}}+P_{\epsilon}$ & $P_{\sigma}-P_{\mu}$ \\
\hline $\mathrm{s}$ & $P_{0}+P_{\psi}+P_{\bar{\psi}}+P_{\epsilon}$ & $-P_{\sigma}-P_{\mu}$ \\
\hline $\mathrm{s}^{\prime}$ & $P_{0}-P_{\psi}-P_{\bar{\psi}}+P_{\epsilon}$ & $-P_{\sigma}+P_{\mu}$ \\
\hline $\mathrm{d} 12$ & $\sqrt{2}\left(P_{0}+P_{\psi}-P_{\bar{\psi}}-P_{\epsilon}\right)$ & 0 \\
\hline $\mathrm{d} 34$ & $\sqrt{2}\left(P_{0}-P_{\psi}+P_{\bar{\psi}}-P_{\epsilon}\right)$ & 0 \\
\hline
\end{tabular}

Table 9. Explicit forms of the defect operators in the fermionic Ising model.

\begin{tabular}{|c|c|c|c|}
\hline & $D_{1}^{\epsilon \epsilon}$ & $D_{1}^{\sigma \sigma}$ & $D_{a}^{\sigma \mu}$ \\
\hline$D_{1}^{\epsilon \epsilon}$ & 1 & & \\
\hline$D_{1}^{\sigma \sigma}$ & $D_{1}^{\sigma \sigma}$ & $\frac{1}{2}\left(1+D_{1}^{\epsilon \epsilon}\right)$ & \\
\hline$D_{a}^{\sigma \mu}$ & $-D_{a}^{\sigma \mu}$ & 0 & $\frac{1}{2}\left(-1+D_{1}^{\epsilon \epsilon}\right)$ \\
\hline
\end{tabular}

Table 10. Interface sewing constraints for an interface between the standard and fermionic Ising models.

\subsection{Interfaces between Ising and fermionic Ising}

As an example, consider interfaces between the bosonic Ising model and the fermionic version. Let us assume that the Ising model is in the upper half plane and the fermionic model in the lower half plane. This means that the possible bulk-defect structure constants are $D_{x}^{i j}$ where $i$ takes values in $\{\epsilon, \sigma\}, j$ takes values in $\{\epsilon, \sigma, \mu\}$ and $x \in\{1, a\}$.

The interface sewing constraints are exactly the appropriate subset of the fermionic defect sewing constraints in table 7 , as given in table 10. There are four solutions to the sewing constraints, which constitute the four one-dimensional representations of the commutative interface classifying algebra and which also form two one-dimensional representations on $\mathbb{C}^{1 \mid 0}$ and one two-dimensional representation on $\mathbb{C}^{1 \mid 1}$ of the interface classifying super-algebra, as in table 11.

\section{Further Virasoro examples}

In this section we will consider a few examples of fermionic minimal models by increasing weight of the generator $G$ as listed in table 1 . The smallest value is $h_{G}=1 / 2$ in the single model $F M(4,3)=\widetilde{F M}(4,3)$ which is the free fermion and was already treated in section 5 .

The next value is $h_{G}=3 / 2$, so that the fermionic theory has super-Virasoro symmetry. There are two such examples, $F M(4,5)$ which is the fermionic tri-critical Ising model (section 6.1) and the non-unitary models $F M(3,8) / \widetilde{F M}(3,8)$ (section 6.2). 


\begin{tabular}{|c|c|c|}
\hline$D^{\epsilon \epsilon}$ & $D^{\sigma \sigma}$ & $D^{\sigma \mu}$ \\
\hline 1 & 1 & 0 \\
\hline 1 & -1 & 0 \\
\hline$-\mathbb{1}$ & 0 & $\mathbb{A}$ \\
\hline
\end{tabular}

Table 11. Solutions to the interface sewing constraints for an interface between the standard and fermionic Ising models.

The $N=1$ superconformal minimal model values of $c$ are $c(p, q)=15 / 2-3 p / q-3 q / p$, parametrised by two integers $p, q$ with $p-q$ even and $p, q \geq 2[25,26]$; we shall denote them generically by $S M(p, q)$. The possible modular invariant partition functions for the unitary models $|p-q|=2$ have been classified in [27] but, as noted there, modular invariance does not fix the partition function uniquely and it is determined only up to a constant which is fixed by the parities of the states with $h=c / 24$.

The final value we consider in any detail is $h_{G}=5 / 2$ in $F M(4,7)$ in section 6.3. This final case was first noted in [28] and a longer list is given in [29] where chiral algebras which extend the Virasoro algebra by a single fermionic field are considered. $F M(4,7)$ is a reduction the $W B(0,2)$ algebra (a.k.a. the fermionic $W B_{2}$ algebra) at a value of $c$ at which the spin 4 field decouples.

These examples in fact all fit into two infinite series of fermionic models with a current of spin $(2 k-1) / 2$, and these are $F M(4,2 k+1)$ and $F M(3,4 k)$. For $k \geq 3$, these two series are all special cases of the fermionic $\mathrm{W}$-algebra $W B(0, k-1)$ (a.k.a. the fermionic $W B_{k-1}$ algebra first introduced in [30]) in which all but the fermionic W-algebra field decouple.

\subsection{Fermionic tri-critical Ising model}

The fermionic tri-critical Ising model is the second in the series of fermionic extensions of the minimal models and some essential data is given in appendix A.2.

\subsubsection{Fermionic TCIM boundary classifying algebra}

The boundary conditions of the tri-critical Ising model in both bosonic and fermionic models have been studied before. For the bosonic case see [31], and for the fermionic case see for example $[13,14]$. Here we will study boundary conditions of the fermionic model via the fermionic classifying algebra.

In the fermionic tri-critical Ising model, there are eight spinless bulk fields which can hence couple to a weight zero boundary field, as in table 12 .

As before, we assume that the only possible weight zero boundary fields are the identity 1 and an odd field $a$, and so the bulk-boundary couplings fields are

$$
B_{1}^{1} \equiv 1, B_{1}^{\epsilon}, B_{1}^{\epsilon^{\prime}}, B_{1}^{\epsilon^{\prime \prime}}, B_{1}^{\sigma}, B_{1}^{\sigma^{\prime}}, B_{a}^{\mu}, B_{a}^{\mu^{\prime}} .
$$

The sewing constraints can again be considered as the relations in an 8 dimensional commutative algebra which also has the form of a non-supercommutative super-algebra with even generators $\left\{1, B^{\epsilon}, B^{\epsilon^{\prime}}, B^{\epsilon^{\prime \prime}}, B^{\sigma}, B^{\sigma^{\prime}}\right\}$ and odd generators $\left\{B^{\mu}, B^{\mu^{\prime}}\right\}$. It is also a graded 


\begin{tabular}{|c|c|c|c|c|c|c|c|c|}
\hline \multirow{3}{*}{$\begin{array}{c}\text { spinless } \\
\text { fields }\end{array}$} & \multicolumn{6}{|c|}{ even } & \multirow{2}{*}{\multicolumn{2}{|c|}{$\begin{array}{c}\text { odd } \\
\mathrm{R}\end{array}$}} \\
\hline & \multicolumn{4}{|c|}{ NS } & \multicolumn{2}{|c|}{$\mathrm{R}$} & & \\
\hline & 1 & $\epsilon$ & $\epsilon^{\prime}$ & $\epsilon^{\prime \prime}$ & $\sigma$ & $\sigma^{\prime}$ & $\mu$ & $\mu^{\prime}$ \\
\hline label & $(1,1)$ & $(3,3)$ & $(1,3)$ & $(3,1)$ & $(2,3)$ & $(2,1)$ & $(2,3)$ & $(2,1)$ \\
\hline$h=\bar{h}$ & 0 & $\frac{1}{10}$ & $\frac{3}{5}$ & $\frac{3}{2}$ & $\frac{3}{80}$ & $\frac{7}{16}$ & $\frac{3}{80}$ & $\frac{7}{16}$ \\
\hline
\end{tabular}

Table 12. Spinless bulk fields in the fermionic tri-critical Ising model.

\begin{tabular}{|c|c|c|}
\hline & $\phi=0$ & $\phi=1$ \\
\hline$\tilde{\phi}=0$ & $B^{1} \equiv 1, B^{\epsilon}, B^{\epsilon^{\prime}}, B^{\epsilon^{\prime \prime}}$ & $B^{\mu}, B^{\mu^{\prime}}$ \\
\hline$\tilde{\phi}=1$ & $B^{\sigma}, B^{\sigma^{\prime}}$ & - \\
\hline
\end{tabular}

Table 13. The bi-grading of the generators of the tri-critical Ising model boundary classifying algebra.

algebra with respect to the spin grading, so it is in fact a bi-graded algebra. The generators are shown along with their gradings $\phi$ and $\tilde{\phi}=\phi+\nu$ in table 13 .

There are 28 sewing constraints for these structure constants which have eight solutions corresponding to the eight one-dimensional representations of the commutative algebra with generators (6.1).

When viewed as super-algebra, the boundary classifying algebra decomposes into four copies of $\mathbb{C}$ and two copies of $C \ell_{1}$, giving six boundary conditions in all. This means the eight one-dimensional representations combine to form 4 one-dimensional representations on $\mathbb{C}^{1 \mid 0}$ and two two-dimensional representations on $\mathbb{C}^{1 \mid 1}$, as shown in table 14 .

The boundary states in the NS and R sectors are given by taking linear combinations determined by table 14 with an overall normalisation,

$$
|a\rangle_{\mathrm{NS}}=\lambda_{a}\left(|0\rangle+B_{1}^{\epsilon}|\epsilon\rangle+B_{1}^{\epsilon^{\prime}}\left|\epsilon^{\prime}\right\rangle+B_{1}^{\epsilon^{\prime \prime}}\left|\epsilon^{\prime \prime}\right\rangle\right), \quad|a\rangle_{\mathrm{R}}=\lambda_{a}\left(B_{1}^{\sigma}|\sigma\rangle+B_{1}^{\sigma^{\prime}}\left|\sigma^{\prime}\right\rangle\right) .
$$

The normalisation is fixed (up to a sign) by the requirement that the number of weight-zero fields is 1 on the boundaries corresponding to one-dimensional representations and 2 on the boundaries corresponding to two-dimensional representations.

\subsubsection{Comparison to $[13,14]$}

There has been a considerable amount of work on boundary conditions of superconformal field theories. Here we compare our results with those of Nepomechie in [14] which discusses boundary states in the tri-critical Ising model $M(4,5)$ and which of these are "supersymmetric". The boundary states discussed in that paper include both NS- and R-sectors and a boundary condition is said to be supersymmetric if the partition function 


\begin{tabular}{|c|ccc|cc|cc|}
\hline boundary & $B^{\epsilon}$ & $B^{\epsilon^{\prime}}$ & $B^{\epsilon^{\prime \prime}}$ & $B^{\sigma}$ & $B^{\sigma^{\prime}}$ & $B^{\mu}$ & $B^{\mu^{\prime}}$ \\
\hline$(1,1) \equiv(-)$ & 1 & 1 & 1 & 1 & 1 & 0 & 0 \\
\hline$(3,1) \equiv(+)$ & 1 & 1 & 1 & -1 & -1 & 0 & 0 \\
\hline$(1,2) \equiv(-0)$ & $-\alpha$ & $-\alpha$ & 1 & $\alpha$ & -1 & 0 & 0 \\
\hline$(1,3) \equiv(0+)$ & $-\alpha$ & $-\alpha$ & 1 & $-\alpha$ & 1 & 0 & 0 \\
\hline$(2,1) \equiv(0)$ & $-\mathbb{1}$ & $\mathbb{1}$ & $-\mathbb{1}$ & 0 & 0 & $\delta \mathbb{A}$ & $-\beta \mathbb{A}$ \\
\hline$(2,2) \equiv(d)$ & $\alpha \mathbb{1}$ & $-\alpha \mathbb{1}$ & $-\mathbb{1}$ & 0 & 0 & $-\gamma \mathbb{A}$ & $\beta \mathbb{A}$ \\
\hline
\end{tabular}

Table 14. The boundary conditions of $F M(4,5)$ at $c=7 / 10 ; \alpha=(3-\sqrt{5}) / 2, \beta=(2 / \sqrt{7})(1+i)$, $\gamma=\sqrt{7}(3-\sqrt{5})(1+i), \delta=2 \sqrt{7}(1+i)$.

on a cylinder is a sum of characters of the super Virasoro algebra, rather than simply a sum of characters of the Virasoro algebra.

Since the fermionic theory $F M(4,5)$ we consider includes the generators of the superVirasoro algebra, the partition function calculated using the overlaps of the boundary states in the NS-sector will always be sums of characters of the super-Virasoro algebra. However, these are not the partition functions discussed in [13] which are instead the average over the two spin structures, that is the average of the overlaps between the NS-sectors and the R-sectors. Since the R-sectors will contribute the supertrace over a super-Virasoro representation, not a trace, any contribution from the R-sector will stop the partition function being a sum of super-Virasoro characters. Hence we see that "supersymmetric" boundary conditions in the sense of [14] correspond to boundary conditions with zero Rsector boundary state, that is boundary conditions for which the algebra of weight zero boundary fields is $C \ell_{1}$; if the weight zero boundary fields are simply $\mathbb{C}$ then the boundary condition is "non-supersymmetric". The latter boundary conditions fall into pairs related by the spin symmetry and which give "supersymmetric" boundary conditions when taken as a superposition.

In this sense we find, as in [14], two "supersymmetric" boundary conditions and four "non-supersymmetric" ones.

\subsubsection{Fermionic TCIM defect classifying algebra}

The defect classifying algebra of $F M(4,5)$ has one generator $D^{i j}$ for each pair of fields $\phi_{i}, \phi_{j}$ whose operator product on the defect includes a field of weight zero. There are 6 such pairs where $i$ and $j$ are both even which generate the defect classifying algebra of the bosonic $M(4,5)$. There are 6 more pairs for which $i$ and $j$ are both odd, but for which $D^{i j}$ is again therefore even. Together these generate the 12 dimensional even subalgebra of $\hat{\mathcal{D}}$. There are further 4 pairs where one of $i$ and $j$ is even and the other odd and these span the four dimensional odd part of $\hat{\mathcal{D}}$, see table 15 . As a vector space, $\hat{\mathcal{D}}$ is equivalent to $\mathbb{C}^{12 \mid 4}$. As a super-algebra, $\hat{\mathcal{D}}$ splits into 8 copies of $\mathbb{C}$ and four copies of $C \ell_{1}$ giving 12 defects in total, twice as many as the purely bosonic defects of $M(4,5)$, with the differences occurring in sectors that are not in $M(4,5)$. 


\begin{tabular}{|c|c|}
\hline Even generators & $D_{1}^{11} \equiv 1, D_{1}^{\epsilon \epsilon}, D_{1}^{\epsilon^{\prime} \epsilon^{\prime}}, D_{1}^{\epsilon^{\prime \prime} \epsilon^{\prime \prime}}, D_{1}^{\sigma \sigma}, D_{1}^{\sigma^{\prime} \sigma^{\prime}}, D_{1}^{\mu \mu}, D_{1}^{\mu^{\prime} \mu^{\prime}}, D_{1}^{G G}, D_{1}^{\bar{G} \bar{G}}, D_{1}^{\psi \psi}, D_{1}^{\bar{\psi} \bar{\psi}}$ \\
\hline Odd generators & $D_{a}^{\sigma \mu}, D_{a}^{\mu \sigma}, D_{a}^{\sigma^{\prime} \mu^{\prime}}, D_{a}^{\mu^{\prime} \sigma^{\prime}}$ \\
\hline
\end{tabular}

Table 15. The generators of the defect classifying algebra of $F M(4,5)$.

\begin{tabular}{|c|ccccccc|}
\hline 2 & $\frac{3}{2}$ & $\frac{25}{32}$ & $\frac{1}{4}$ & $-\frac{3}{32}$ & $-\frac{1}{4}$ & $-\frac{7}{32}$ & 0 \\
1 & 0 & $-\frac{7}{32}$ & $-\frac{1}{4}$ & $-\frac{3}{32}$ & $\frac{1}{4}$ & $\frac{25}{32}$ & $\frac{3}{2}$ \\
\hline$r / s$ & 1 & 2 & 3 & 4 & 5 & 6 & 7 \\
\hline
\end{tabular}

Table 16. The representations of the Virasoro algebra at $c=-21 / 4$.

If we restrict $i$ and $j$ to the NS sector alone, the algebra is instead equivalent to $\mathbb{C}^{8 \mid 0}$, i.e. it is purely even and the corresponding eight solutions are formally the same as those found in [12].

Up to now, the question has been entirely one of calculating the bulk-defect structure constants. The final step is to fix the normalisation of the defect operators (up to a sign) to reproduce the correct counting of weight zero fields from the torus expectation value of the defect operator which is easily done.

\subsubsection{Fermionic TCIM interface classifying algebra}

The only interface we can consider is that between $M(4,5)$ and $F M(4,5)$. Since the fields in $M(4,5)$ form a subalgebra of the fields in $F M(4,5)$, the interface classifying algebra is a subalgebra of the defect classifying algebra of $F M(4,5)$. From table 15, there are 6 surviving even generators and 2 surviving odd generators. This means the interface classifying algebra is $\mathbb{C}^{6 / 2}$ as a super-vector space, splitting into four copies of $\mathbb{C}$ and two copies of $C \ell_{1}$ as a graded algebra, giving 6 interfaces in total.

\section{2 $F M(3,8) / \widetilde{F M}(3,8)$, the supersymmetric Lee-Yang model}

The case of $c=-21 / 4$ is the first where there is a half-integer spin simple current allowing the extension of a bosonic Virasoro minimal model to a fermionic model and there are also two bulk invariants. There is the diagonal model $M\left(A_{2}, A_{7}\right)$ and the Dinvariant $M\left(A_{2}, D_{5}\right)$.

The representations and their weights are given in table 16. As usual the Kac-table includes two copies of each representation and in this case we remove the degeneracy by considering only the representations of type $(1, s)$.

The value $c=-21 / 4$ corresponds to $S M(2,8)$ and has been looked at before, see e.g. [32-34]. It has been identified as the supersymmetric Lee-Yang model. The issue of different models at the same central charge does not seem to have been considered in these works - they assume that the bosonic projection is the diagonal invariant $M(3,8)$, and hence in our language identify the superconformal theory as $F M(3,8)$. 


\begin{tabular}{|c|ccc|}
\hline$(r, s)$ & $h_{r, s}$ & $N S / R$ & $\hat{M}_{r, s}$ \\
\hline$(1,1)$ & 0 & $N S$ & $\hat{M}_{1,1}=M_{1,1} \oplus M_{1,7}$ \\
\hline$(1,2)$ & $-\frac{7}{32}$ & $R$ & $\hat{M}_{1,2}=M_{1,2} \oplus M_{1,6}$ \\
\hline$(1,3)$ & $-\frac{1}{4}$ & $N S$ & $\hat{M}_{1,3}=M_{1,3} \oplus M_{1,5}$ \\
\hline$(1,4)$ & $-\frac{3}{32}$ & $R$ & $\hat{M}_{1,4}=M_{1,4}$ \\
\hline
\end{tabular}

Table 17. The representations of the super-Virasoro algebra at $c=-21 / 4$.

\begin{tabular}{|c|c|c|c|}
\hline \multicolumn{4}{|c|}{ A invariant } \\
\hline even, NS & even, R & odd, NS & odd, R \\
\hline$\phi_{(1, a)}^{e}$ & $\phi_{(1, a)}^{e}$ & $\phi_{(1, a)}^{o}$ & $\phi_{(1, a)}^{o}$ \\
\hline$(1,1)(1,1)$ & $(1,2)(1,2)$ & $(1,1)(1,7)$ & $(1,2)(1,6)$ \\
$(1,3)(1,3)$ & $(1,4)(1,4)$ & $(1,7)(1,1)$ & $(1,4)(1,4)$ \\
$(1,5)(1,5)$ & $(1,6)(1,6)$ & $(1,3)(1,5)$ & $(1,6)(1,2)$ \\
$(1,7)(1,7)$ & & $(1,5)(1,3)$ & \\
\hline
\end{tabular}

\begin{tabular}{|c|c|c|c|}
\hline \multicolumn{4}{|c|}{ D invariant } \\
\hline even, NS & even, R & odd, NS & odd, R \\
\hline$\phi_{(1, a)}^{u}$ & $\phi_{(1, a)}^{s}$ & $\phi_{(1, a)}^{s}$ & $\phi_{(1, a)}^{u}$ \\
\hline$(1,1)(1,1)$ & $(1,2)(1,6)$ & $(1,1)(1,7)$ & $(1,2)(1,2)$ \\
$(1,3)(1,3)$ & $(1,4)(1,4)$ & $(1,7)(1,1)$ & $(1,4)(1,4)$ \\
$(1,5)(1,5)$ & $(1,6)(1,2)$ & $(1,3)(1,5)$ & $(1,6)(1,6)$ \\
$(1,7)(1,7)$ & & $(1,5)(1,3)$ & \\
\hline
\end{tabular}

Table 18. The field content of the $A$ and $D$ invariants of $M_{3,8}$ (even part of the table) and their fermionic extensions (even and odd part). The fermionic extensions differ only in their Ramond sector parity. Listed are the parity and spin grade, the notation of the primary bulk field used in section 4, and the Kac-label of the left/right representation of that field.

At $c=-21 / 4$, there are 4 relevant representations of the super-Virasoro algebra with labels $(r, s)$, shown in table 17. Since the Virasoro algebra is a subalgebra, each representation $\hat{M}_{r, s}$ of SVir decomposes into a sum of one or more representations $M_{r, s}$ of Vir and this information is included in this table along with their sector.

As there are two different invariants of the Virasoro algebra, there are two different fermionic extensions. These are related by parity shift in the Ramond sector and are the fermionic models $F M(3,8)$ and $\widetilde{F M}(3,8)$ from section 4 . Their field content is given in table 18.

The representation content in the even and odd Ramond sector of $F M(3,8)$ and $\widetilde{F M}(3,8)$ now differs, and so these models cannot be graded-isomorphic. From the point of view of the super-Virasoro algebra, the parity of the Ramond sector ground state has changed between these two models, cf. table 17, where the R-ground state has label $(1,2)$.

The structure of the boundary classifying algebras also differs in the two models. According to the general theory in section 3.3, there are equal numbers of bulk-boundary structure constants in the two models, but the parities are different. In $F M(3,8)$, where the Ramond ground state is even, there are 7 even and 1 odd generators. This leads to a classifying algebra which is $\mathbb{C}^{7 \mid 1}$ as a super-vector space and which decomposes into 6 copies 


\begin{tabular}{|c|c|c|}
\hline & $\phi=0$ & $\phi=1$ \\
\hline$\tilde{\phi}=0$ & $B^{(1,1), e}, B^{(1,3), e}, B^{(1,5), e}, B^{(1,7), e}$ & $B^{(1,4), o}$ \\
\hline$\tilde{\phi}=1$ & $B^{(1,2), e}, B^{(1,4), e}, B^{(1,6), e}$ & - \\
\hline
\end{tabular}

Table 19. The bi-grading of the generators of the boundary classifying algebras of $F M(3,8)$ (parity $\phi)$ and $\widetilde{F M}(3,8)$ (parity $\tilde{\phi})$, with the identification $B^{(1, a), u}=B^{(1, a), e}, B^{(1,4), s}=i B^{(1,4), o}$, cf. $(3.20)$.

of $\mathbb{C}$ and 1 copy of $C \ell_{1}$ giving seven fundamental boundary conditions. From the point of view of the superconformal algebra, this means there is 1 "supersymmetric" boundary condition in the sense of [13] and 6 "non-supersymmetric" ones.

In $\widetilde{F M}(3,8)$, where the Ramond ground state is odd, there are 5 even and 3 odd generators, leading to a classifying algebra which is $\mathbb{C}^{5 \mid 3}$ as a super-vector space and which decomposes into 2 copies of $\mathbb{C}$ and 3 copies of $C \ell_{1}$ giving five fundamental boundary conditions, 3 of which are "supersymmetric" and 2 are not.

As expected, the boundary conditions of $F M(3,8)$ are in 1-1 correspondence with the 7 boundary conditions of $M\left(A_{2}, A_{7}\right)$, and those of $\widetilde{F M}(3,8)$ are in 1-1 correspondence with the 5 boundary conditions of $M\left(A_{2}, D_{5}\right)$ (see [35] for the boundary conditions of minimal models).

The two boundary classifying algebras can be made identical [as ungraded algebras] by a suitable rescaling of the fields in $\widetilde{F M}(3,8)$ (or equivalently of the generators of the algebra). The generators and their gradings are shown in table 19 together with the rescaling of the generators that makes the algebras identical.

There are now three different fermionic defect classifying algebras, classifying the defects in $F M(3,8)$, defects in $\widetilde{F M}(3,8)$ and interfaces between $F M(3,8)$ and $\widetilde{F M}(3,8)$. Again, according to the general theory in section 3.3, these can be made equal as ungraded algebras by a simple rescaling of the generators by phases, but they are not all equivalent as graded algebras. The two defect classifying algebras have the same gradings, but the interface algebra has a different grading. These are shown in table 20. The two defect classifying algebras are $\mathbb{C}^{14 \mid 2}$ as vector spaces, decomposing into 12 copies of $\mathbb{C}$ and two copies of $C \ell_{1}$ as graded algebras, giving 14 defects in each of these two theories. The interface algebra is $\mathbb{C}^{10 \mid 6}$ as a vector space and 4 copies $\mathbb{C}$ and 6 copies of $C \ell_{1}$ as an algebra giving 10 interfaces between the two theories. When we descend to the bosonic $A$ and $D$ theories, the defects/interfaces in the fermionic theories are identified in pairs (as in subsection 6.1.3, the pairs only differ in sectors that are not in the bosonic theories) and give 7 defects in the each of the $A$ and $D$ theories and 5 interfaces between the $A$ and $D$ theories, in agreement with the computation in terms of traces of products of bulk modular invariant matrices, see [24] and [36, remark 5.19].

\subsection{The $F M(4,7)=W B(0,2)_{5,7}$ example}

Finally, the only fermionic minimal model to have a weight $5 / 2$ current is $F M(4,7)$ with central charge $c=-13 / 14$. This turns out to be a restriction of the "fermionic" Walgebra $W B(0,2)$. This algebra, also known as the "fermionic" $W B_{2}$ algebra, extends 


\begin{tabular}{|c|c|c|}
\hline & $\phi=0$ & $\phi=1$ \\
\hline$\tilde{\phi}=0$ & $D^{[(1,1), e][(1,1), e]}, D^{[(1,3), e][(1,3), e]}, D^{[(1,5), e][(1,5), e]}, D^{[(1,7), e][(1,7), e]}$, & $D^{[(1,4), e][(1,4), o]}$, \\
& $D^{[(1,1), o][(1,1), o]}, D^{[(1,3), o][(1,3), o]}, D^{[(1,5), o][(1,5), o]}, D^{[(1,7), o][(1,7), o]}$ & $D^{[(1,4), o][(1,4), e]}$ \\
\hline$\tilde{\phi}=1$ & $D^{[(1,2), e][(1,2), e]}, D^{[(1,4), e][(1,4), e]}, D^{[(1,6), e][(1,6), e]}$, & - \\
& $D^{[(1,2), o][(1,2), o]}, D^{[(1,4), o][(1,4), o]}, D^{[(1,6), o][(1,6), o]}$ & \\
\hline
\end{tabular}

Table 20. The bi-grading of the generators of the defect and interface classifying algebras of $F M(3,8)$ and $\widetilde{F M}(3,8)$. The parity of a given generator in either of the two defect algebras is called $\phi$, and in the interface algebra it is called $\tilde{\phi}$, with the identifications used in (3.22).

the Virasoro algebra by primary fields of weights 4 and $5 / 2$. Its structure constants were worked out explicitly in [37] and it can be seen that the field of weight 4 decouples from the algebra at this value of $c$. The minimal model of this algebra has 6 representations labelled by $\left[r s ; r^{\prime} s^{\prime}\right]$ where $r, s, r^{\prime}, s^{\prime} \geq 1,2 r+s \leq 3$ and $2 r^{\prime}+s^{\prime} \leq 5$. Three of these are irreducible as Virasoro algebra representations and in the Ramond sector and give the three R sector representations of $F M(4,7)$; three are reducible, splitting each into two Virasoro algebra representations and these together are the six NS sector representations of $F M(4,7)$. The rest of the analysis is straightforward - there are 9 boundary conditions of $F M(4,7)$ of which three are invariant under the W-algebra automorphism $W \rightarrow-W$ and three are related in pairs. This is exactly as in the fermionic TCIM, where there are two "supersymmetric" boundary conditions and two pairs which are related by $G \rightarrow-G$.

\section{Conclusions}

We have defined fermionic conformal field theories and their classifying algebras, defined fermionic extensions of the Virasoro minimal models, found explicit expressions for all the bulk structure constants (of both Neveu-Schwarz and Ramond fields) of these models, and have given numerous examples in these cases.

We have found that it is natural for certain boundary conditions and defects to support a weight zero fermionic field which has up to now been introduced in an ad hoc manner.

We have also found that there is a natural parity-shift operation which can relate different theories. On the one hand, this relates bosonic theories as the projections of parity-shifted fermionic theories, and on the other hand this means that there are hitherto unconsidered fermionic theories to be looked at.

There are quite a few questions that are unresolved and which suggest new lines of enquiry.

Firstly, we showed that the full set of bulk-boundary structure constants in the Ising model defined an algebra, not just the couplings to boundary fields of weight zero. This seems to merit further investigation, even in the purely bosonic case. 
Secondly, we showed that the fermionic extensions of the $A$ - and $D$ - invariant minimal models are related by a parity-shift operation. This leads one to wonder if one can define extensions in other cases in which the extended algebra would be bosonic, such as the $A$ - and $D$ - invariants of $M(5,6)$, namely the tetra-critical Ising model and the 3 -state Potts model respectively. It would also be interesting to investigate possible fermionic extensions of the exceptional invariants; one of these, $M\left(A_{4}, E_{6}\right)$, is a product theory $M\left(A_{1}, A_{4}\right) \times M\left(A_{2}, A_{3}\right)=M(2,5) \times M(3,4)$ [38], with the obvious proposal that $F M\left(A_{4}, E_{6}\right)=M(2,5) \times F M(3,4)$. It would be good to have a general understanding of these models.

It would also be good to give the structure constants for the field theories on the boundaries, defects and interfaces that we have found which would be a necessary first step to discuss their perturbations and the resulting renormalisation group flows.

In [39], the moduli space of $c=1$ CFTs with fermions was investigated (see figure 2 there), it would be interesting to look at this problem from our perspective.

Finally, one consequence of our construction relating $F M(p, q)$ to $\widetilde{F M}(p, q)$ is that when these are also superconformal field theories, their partition functions differ in the sign of the "bottom component" of the super-partition function, that is $Z_{R R}=\operatorname{TR}_{R}\left((-1)^{F}\right)$ which is the trace of $(-1)^{F}$ on the highest weight space of the Ramond fields [40]. This cannot be determined on the grounds of modular invariance. We have found that in our construction, $Z_{R R}=+1$ for $F M(3,8)$ and $Z_{R R}=-1$ for $\widetilde{F M}(3,8)$ which are hence two inequivalent superconformal field theories at $c=-21 / 4$. It is a curious fact that this implies that in this model the Virasoro characters satisfy $\chi_{1,2}=\chi_{1,6}+1$, as is easily checked from the character formulae in [21]. The value $Z_{R R}=0$ for the TCIM was already observed in [40]. This leaves open now the question of investigating the superconformal models in which this parity-shift relates two inequivalent field theories, and how this might affect previous results on boundary conditions, boundary perturbations, etc.

\section{Acknowledgments}

We would like to thank L. Szegedy for helpful discussions and careful reading of the manuscript. GW thanks I. Makabe for many discussions on boundary conditions in superconformal field theories, P. Mathieu for discussions on superconformal models in general, as well as S. Majid, A. Recknagel and S. Wood for further useful discussions. IR thanks the Department of Mathematics at King's College London for hospitality during a sabbatical in the first half of 2019 where this research was completed. IR is partially supported by the Deutsche Forschungsgemeinschaft via the Research Training Group RTG 1670 and the Cluster of Excellence EXC 2121.

\section{A Data for the Ising and tri-critical Ising models}

\section{A.1 Ising data}

We list here the data for the Ising model that is used in section 5 . The representations $(1,1),(1,2),(1,3)$ with conformal weights $0, \frac{1}{16}$ and $\frac{1}{2}$ are denoted $1, \sigma$ and $\epsilon$. 
The bulk field content is as follows, giving both the conventional name from the freefermionic extension of the Ising model and the names following the conventions of section 4.1:

\begin{tabular}{|c|c|c|c|}
\hline $\begin{array}{c}\text { Even } \\
(\bar{r}, \bar{s}) ; \bar{h}\end{array}$ & \multicolumn{3}{|c|}{$(r, s) ; h$} \\
\hline$(1,1) ; 0$ & $1 \equiv \phi_{1}^{e}$ & - & - \\
$(1,2) ; \frac{1}{16}$ & - & $\sigma \equiv \phi_{\sigma}^{e}$ & - \\
$(1,3) ; \frac{1}{2}$ & - & - & $\epsilon \equiv \phi_{\epsilon}^{e}$ \\
\hline
\end{tabular}

\begin{tabular}{|c|c|c|c|}
\hline Odd & \multicolumn{3}{|c|}{$(r, s) ; h$} \\
$(\bar{r}, \bar{s}) ; \bar{h}$ & $(1,1) ; 0$ & $(1,2) ; \frac{1}{16}$ & $(1,3) ; \frac{1}{2}$ \\
\hline$(1,1) ; 0$ & - & - & $\psi \equiv \phi_{\epsilon}^{o}$ \\
$(1,2) ; \frac{1}{16}$ & - & $\mu \equiv \phi_{\sigma}^{o}$ & - \\
$(1,3) ; \frac{1}{2}$ & $\bar{\psi} \equiv \phi_{1}^{o}$ & - & - \\
\hline
\end{tabular}

The values for the normalisation constants chosen in (4.5) to get the structure constants in section 5.1 are

$$
\lambda_{1}^{e}=1, \quad \lambda_{\epsilon}^{e}=1, \quad \lambda_{\sigma}^{e}=2^{1 / 4}, \quad \lambda_{1}^{o}=1, \quad \lambda_{\epsilon}^{o}=i, \quad \lambda_{\sigma}^{o}=e^{\pi i / 4} 2^{-1 / 4} .
$$

The F-matrix entries we need are:

$$
\begin{array}{llll}
F_{00}\left[\begin{array}{cc}
\sigma & \sigma \\
\sigma & \sigma
\end{array}\right]=\frac{1}{\sqrt{2}} & F_{0 \epsilon}\left[\begin{array}{cc}
\sigma & \sigma \\
\sigma & \sigma
\end{array}\right]=\frac{1}{2 \sqrt{2}} & F_{\epsilon 0}\left[\begin{array}{cc}
\sigma & \sigma \\
\sigma & \sigma
\end{array}\right]=\sqrt{2} & F_{\epsilon \epsilon}\left[\begin{array}{cc}
\sigma & \sigma \\
\sigma & \sigma
\end{array}\right]=\frac{-1}{\sqrt{2}} \\
F_{0 \sigma}\left[\begin{array}{cc}
\sigma & \epsilon \\
\sigma & \epsilon
\end{array}\right]=\frac{1}{2} & F_{\sigma 0}\left[\begin{array}{cc}
\epsilon & \epsilon \\
\sigma & \sigma
\end{array}\right]=2 & F_{\sigma \sigma}\left[\begin{array}{cc}
\epsilon & \sigma \\
\sigma & \epsilon
\end{array}\right]=-1 & F_{00}\left[\begin{array}{cc}
\epsilon & \epsilon \\
\epsilon & \epsilon
\end{array}\right]=1 .
\end{array}
$$

In the discussion of boundary states and defects we make use of the modular transformations of Virasoro characters, with $q=\exp (2 \pi i \tau)$ and $\tilde{q}=\exp (-2 \pi i / \tau)$,

$$
\begin{aligned}
& \chi_{0}(\tilde{q})=\frac{1}{2} \chi_{0}(q)+\frac{1}{\sqrt{2}} \chi_{\sigma}(q)+\frac{1}{2} \chi_{\epsilon}(q), \\
& \chi_{\sigma}(\tilde{q})=\frac{1}{\sqrt{2}} \chi_{0}(q)-\frac{1}{\sqrt{2}} \chi_{\epsilon}(q), \\
& \chi_{\epsilon}(\tilde{q})=\frac{1}{2} \chi_{0}(q)-\frac{1}{\sqrt{2}} \chi_{\sigma}(q)+\frac{1}{2} \chi_{\epsilon}(q) .
\end{aligned}
$$

\section{A.2 Tri-critical Ising data}

The tri-critical Ising model is the Virasoro minimal model $M(4,5)$. The bulk model is described in [41] and the conformal boundary conditions in [31].

In table 21 we list the even and odd sectors in the same manner as for the Ising model, using mostly the naming conventions of [41] and [42] [43] use $t$ instead of $\epsilon^{\prime}$ ). The even sector is the field content of the bosonic tri-critical Ising model. Note that the field $G$ is conventionally normalised to $\langle G \mid G\rangle=3 c / 2=21 / 20$ (as opposed to our $\langle G \mid G\rangle=1$ ) and so we only have $G \propto \phi_{1}^{o}$. Likewise, $\epsilon^{\prime \prime} \propto i G \bar{G}$.

As pointed out in [43], the tri-critical Ising model is related to the first non-trivial unitary superconformal minimal model $\operatorname{SM}(3,5)$ which is the associated fermionic model. There are four unitary highest weight representations $\hat{M}_{r, s}$ of the superconformal algebra at $c=7 / 10$. These each decompose as a finite sum of Virasoro representations $M_{r, s}$ as shown in table 22 . 


\begin{tabular}{|c|c|c|c|c|c|c|}
\hline Even & \multicolumn{6}{|c|}{$(r, s) ; h$} \\
$(\bar{r}, \bar{s}) ; \bar{h}$ & $(1,1) ; 0$ & $(3,1) ; \frac{3}{2}$ & $(2,1) ; \frac{7}{16}$ & $(2,3) ; \frac{3}{80}$ & $(1,3) ; \frac{3}{5}$ & $(3,3) ; \frac{1}{10}$ \\
\hline$(1,1) ; 0$ & $1 \equiv \phi_{1}^{e}$ & - & - & - & - & - \\
$(3,1) ; \frac{3}{2}$ & - & $\epsilon^{\prime \prime} \equiv \phi_{\epsilon^{\prime \prime}}^{e}$ & - & - & - & - \\
$(2,1) ; \frac{7}{16}$ & - & - & $\sigma^{\prime} \equiv \phi_{\sigma^{\prime}}^{e}$ & - & - & - \\
$(2,3) ; \frac{3}{80}$ & - & - & - & $\sigma \equiv \phi_{\sigma}^{e}$ & - & - \\
$(1,3) ; \frac{3}{5}$ & - & - & - & - & $\epsilon^{\prime} \equiv \phi_{\epsilon^{\prime}}^{e}$ & - \\
$(3,3) ; \frac{1}{10}$ & - & - & - & - & - & $\epsilon \equiv \phi_{\epsilon}^{e}$ \\
\hline
\end{tabular}

\begin{tabular}{|c|c|c|c|c|c|c|}
\hline Odd & \multicolumn{6}{|c|}{$(r, s) ; h$} \\
$(\bar{r}, \bar{s}) ; \bar{h}$ & $(1,1) ; 0$ & $(3,1) ; \frac{3}{2}$ & $(2,1) ; \frac{7}{16}$ & $(2,3) ; \frac{3}{80}$ & $(1,3) ; \frac{3}{5}$ & $(3,3) ; \frac{1}{10}$ \\
\hline$(1,1) ; 0$ & - & $G \propto \phi_{\epsilon^{\prime \prime}}^{o}$ & - & - & - & - \\
$(3,1) ; \frac{3}{2}$ & $\bar{G} \propto \phi_{1}^{o}$ & - & - & - & - & \\
$(2,1) ; \frac{7}{16}$ & - & - & $\mu^{\prime} \equiv \phi_{\sigma^{\prime}}^{o}$ & - & - & - \\
$(2,3) ; \frac{3}{80}$ & - & - & - & $\mu \equiv \phi_{\sigma}^{o}$ & - & - \\
$(1,3) ; \frac{3}{5}$ & - & - & - & - & - & $\bar{\psi} \equiv \phi_{\epsilon}^{o}$ \\
$(3,3) ; \frac{1}{10}$ & - & - & - & - & $\psi \equiv \phi_{\epsilon^{\prime}}^{o}$ & - \\
\hline
\end{tabular}

Table 21. The odd and even sectors of the bulk tri-critical Ising model.

\begin{tabular}{|c|ccc|}
\hline$(r, s)$ & $h_{r, s}$ & $N S / R$ & $\hat{M}_{r, s}$ \\
\hline$(1,1)$ & 0 & $N S$ & $\hat{M}_{1,1}=M_{1,1} \oplus M_{3,1}$ \\
\hline$(1,2)$ & $\frac{7}{16}$ & $R$ & $\hat{M}_{1,2}=M_{1,2}$ \\
\hline$(1,3)$ & $\frac{1}{10}$ & $N S$ & $\hat{M}_{1,3}=M_{1,3} \oplus M_{3,3}$ \\
\hline$(1,4)$ & $\frac{3}{80}$ & $R$ & $\hat{M}_{1,4}=M_{1,4}$ \\
\hline
\end{tabular}

Table 22. The representations of the super-Virasoro algebra at $c=7 / 10$. 
Open Access. This article is distributed under the terms of the Creative Commons Attribution License (CC-BY 4.0), which permits any use, distribution and reproduction in any medium, provided the original author(s) and source are credited.

\section{References}

[1] S. Novak and I. Runkel, Spin from defects in two-dimensional quantum field theory, arXiv: 1506.07547 [INSPIRE].

[2] I. Runkel, L. Szegedy and G.M.T. Watts, in preparation.

[3] J. Fuchs and C. Schweigert, A Classifying algebra for boundary conditions, Phys. Lett. B 414 (1997) 251 [hep-th/9708141] [INSPIRE].

[4] J. Fuchs, C. Schweigert and C. Stigner, The Classifying algebra for defects, Nucl. Phys. B 843 (2011) 673 [arXiv: 1007.0401] [INSPIRE].

[5] S. Ghoshal and A.B. Zamolodchikov, Boundary $S$ matrix and boundary state in two-dimensional integrable quantum field theory, Int. J. Mod. Phys. A 9 (1994) 3841 [Erratum ibid. A 9 (1994) 4353] [hep-th/9306002] [InSPIRE].

[6] R. Chatterjee and A.B. Zamolodchikov, Local magnetization in critical Ising model with boundary magnetic field, Mod. Phys. Lett. A 9 (1994) 2227 [hep-th/9311165] [INSPIRE].

[7] G.Z. Tóth, A Study of truncation effects in boundary flows of the Ising model on the strip, J. Stat. Mech. 0704 (2007) P04005 [hep-th/0612256] [INSPIRE].

[8] A. Konechny, Critical Ising Model with Boundary Magnetic Field: RG Interface and Effective Hamiltonians, JHEP 04 (2019) 001 [arXiv: 1811.07599] [INSPIRE].

[9] A. Cappelli, C. Itzykson and J.B. Zuber, The ADE Classification of Minimal and A1(1) Conformal Invariant Theories, Commun. Math. Phys. 113 (1987) 1 [InSPIRE].

[10] I. Runkel, Boundary structure constants for the A series Virasoro minimal models, Nucl. Phys. B 549 (1999) 563 [hep-th/9811178] [INSPIRE].

[11] I. Runkel, Structure constants for the D series Virasoro minimal models, Nucl. Phys. B 579 (2000) 561 [hep-th/9908046] [INSPIRE].

[12] I. Makabe and G.M.T. Watts, Defects in the Tri-critical Ising model, JHEP 09 (2017) 013 [arXiv: 1703.09148] [INSPIRE].

[13] R.I. Nepomechie, Consistent superconformal boundary states, J. Phys. A 34 (2001) 6509 [hep-th/0102010] [INSPIRE].

[14] R.I. Nepomechie, Supersymmetry in the boundary tricritical Ising field theory, Int. J. Mod. Phys. A 17 (2002) 3809 [Erratum ibid. A 18 (2003) 2729] [hep-th/0203123] [INSPIRE].

[15] S. Novak and I. Runkel, State sum construction of two-dimensional topological quantum field theories on spin surfaces, J. Knot Theor. Ramifications 24 (2015) 1550028 [arXiv: 1402.2839] [INSPIRE].

[16] D.C. Lewellen, Sewing constraints for conformal field theories on surfaces with boundaries, Nucl. Phys. B 372 (1992) 654 [inSPIRE].

[17] J.L. Cardy and D.C. Lewellen, Bulk and boundary operators in conformal field theory, Phys. Lett. B 259 (1991) 274 [INSPIRE]. 
[18] J. Fuchs, C. Schweigert and C. Stigner, The Three-dimensional origin of the classifying algebra, Nucl. Phys. B 824 (2010) 333 [arXiv:0907.0685] [InSPIRE].

[19] T. Józefiak, Semisimple superalgebras, in Algebra Some Current Trends, proceedings of the 5th National School in Algebra, Varna, Bulgaria, 24 September-4 October 1986, L.L. Avramov and K.B. Tchakerian eds., Springer, Lect. Notes Math. 1352 (1988) 96.

[20] A. Recknagel, Permutation branes, JHEP 04 (2003) 041 [hep-th/0208119] [INSPIRE].

[21] P. Di Francesco, P. Mathieu and D. Sénéchal, Conformal Field Theory, Springer-Verlag, New York U.S.A. (1997).

[22] P.B. Smith and D. Tong, Boundary States for Chiral Symmetries in Two Dimensions, arXiv: 1912.01602 [INSPIRE].

[23] M. Oshikawa and I. Affleck, Boundary conformal field theory approach to the critical two-dimensional Ising model with a defect line, Nucl. Phys. B 495 (1997) 533 [cond-mat/9612187] [INSPIRE].

[24] V.B. Petkova and J.B. Zuber, Generalized twisted partition functions, Phys. Lett. B 504 (2001) 157 [hep-th/0011021] [INSPIRE].

[25] M.A. Bershadsky, V.G. Knizhnik and M.G. Teitelman, Superconformal Symmetry in Two-Dimensions, Phys. Lett. B 151 (1985) 31 [INSPIRE].

[26] O. Blondeau-Fournier, P. Mathieu, D. Ridout and S. Wood, Superconformal minimal models and admissible Jack polynomials, Adv. Math. 314 (2017) 71 [arXiv:1606.04187] [INSPIRE].

[27] A. Cappelli, Modular Invariant Partition Functions of Superconformal Theories, Phys. Lett. B 185 (1987) 82 [inSPIRE].

[28] A.B. Zamolodchikov, Infinite Additional Symmetries in Two-Dimensional Conformal Quantum Field Theory, Theor. Math. Phys. 65 (1985) 1205 [INSPIRE].

[29] R. Blumenhagen, M. Flohr, A. Kliem, W. Nahm, A. Recknagel and R. Varnhagen, $W$ algebras with two and three generators, Nucl. Phys. B 361 (1991) 255 [INSPIRE].

[30] S.L Lukaynov and V.A. Fateev, Additional Symmetries and Exactly Soluble models in two-dimensional conformal field theory, Sov. Sci. Rev. Phys. A 15 (1990) 1.

[31] I. Affleck, Edge critical behavior of the two-dimensional tricritical Ising model, J. Phys. A 33 (2000) 6473 [cond-mat/0005286] [INSPIRE].

[32] K. Schoutens, Supersymmetry and Factorizable Scattering, Nucl. Phys. B 344 (1990) 665 [INSPIRE].

[33] C.-r. Ahn and R.I. Nepomechie, The Scaling supersymmetric Yang-Lee model with boundary, Nucl. Phys. B 594 (2001) 660 [hep-th/0009250] [INSPIRE].

[34] M. Kormos, Boundary renormalisation group flows of the supersymmetric Lee-Yang model and its extensions, Nucl. Phys. B 772 (2007) 227 [hep-th/0701061] [INSPIRE].

[35] R.E. Behrend, P.A. Pearce, V.B. Petkova and J.B. Zuber, Boundary conditions in rational conformal field theories, Nucl. Phys. B 570 (2000) 525 [Nucl. Phys. B 579 (2000) 707] [hep-th/9908036] [INSPIRE].

[36] J. Fuchs, I. Runkel and C. Schweigert, TFT construction of RCFT correlators 1. Partition functions, Nucl. Phys. B 646 (2002) 353 [hep-th/0204148] [INSPIRE]. 
[37] J.M. Figueroa-O'Farrill, S. Schrans and K. Thielemans, On the Casimir algebra of B(2), Phys. Lett. B 263 (1991) 378 [INSPIRE].

[38] T. Quella, I. Runkel and G.M.T. Watts, Reflection and transmission for conformal defects, JHEP 04 (2007) 095 [hep-th/0611296] [INSPIRE].

[39] A. Karch, D. Tong and C. Turner, A Web of $2 d$ Dualities: $\mathbf{Z}_{2}$ Gauge Fields and Arf Invariants, SciPost Phys. 7 (2019) 007 [arXiv: 1902.05550] [INSPIRE].

[40] J.D. Cohn and D. Friedan, Super Characters and Chiral Asymmetry in Superconformal Field Theory, Nucl. Phys. B 296 (1988) 779 [inSPIRE].

[41] M. Lässig, G. Mussardo and J.L. Cardy, The scaling region of the tricritical Ising model in two-dimensions, Nucl. Phys. B 348 (1991) 591 [INSPIRE].

[42] L. Chim, Boundary S matrix for the tricritical Ising model, Int. J. Mod. Phys. A 11 (1996) 4491 [hep-th/9510008] [INSPIRE].

[43] D. Friedan, Z.-a. Qiu and S.H. Shenker, Superconformal Invariance in Two-Dimensions and the Tricritical Ising Model, Phys. Lett. B 151 (1985) 37 [INSPIRE]. 